\title{
Spatial methods for infectious disease outbreak investigations: systematic literature review
}

CM Smith ${ }^{1}$, SC Le Comber ${ }^{2}$, H Fry ${ }^{3}$, M Bull ${ }^{4}$, S Leach ${ }^{4}$, AC Hayward ${ }^{1}$

1. UCL Department of Infectious Disease Informatics, Farr Institute of Health Informatics Research, University College London, London, United Kingdom

2. School of Biological and Chemical Sciences, Queen Mary University of London, London, United Kingdom

3. Centre for Advanced Spatial Analysis, University College London, London, United Kingdom

4. Emergency Response Department Science and Technology, Public Health England, Porton Down, United Kingdom

Correspondence: Catherine Smith (catherine.smith.13@ucl.ac.uk)

Citation style for this article:

Smith CM, Le Comber SC, Fry H, Bull M, Leach S, Hayward AC. Spatial methods for infectious disease outbreak investigations: systematic literature review. Euro Surveill. 2015;20(39):pii=30026. DOI: http://dx.doi.org/10.2807/1560-7917.ES.2015.20.39.30026

Article submitted on 19 February 2015 / accepted on 02 September 2015 / published on 01 October 2015

Investigations of infectious disease outbreaks are conventionally framed in terms of person, time and place. Although geographic information systems have increased the range of tools available, spatial analyses are used relatively infrequently. We conducted a systematic review of published reports of outbreak investigations worldwide to estimate the prevalence of spatial methods, describe the techniques applied and explore their utility. We identified 80 reports using spatial methods published between 1979 and 2013, ca $0.4 \%$ of the total number of published outbreaks. Environmental or waterborne infections were the most commonly investigated, and most reports were from the United Kingdom. A range of techniques were used, including simple dot maps, cluster analyses and modelling approaches. Spatial tools were usefully applied throughout investigations, from initial confirmation of the outbreak to describing and analysing cases and communicating findings. They provided valuable insights that led to public health actions, but there is scope for much wider implementation and development of new methods.

\section{Introduction}

Detecting and responding to outbreaks of infectious diseases is a key role of front-line public health organisations [1]. The primary reason for conducting an investigation into an outbreak is prevention of further cases through control measures, while other motivations include addressing public or political concerns, evaluating health programmes and advancing understanding of the disease [2]. Investigations are usually cross-agency exercises and conventionally involve examination of the outbreak in terms of person, time and place.

John Snow famously demonstrated the power of plotting the spatial locations of individuals affected in an outbreak [3]. His map of cholera cases in London in 1854 showed a clear pattern that implicated a water pump as the likely source of the illness. Today, guidelines for investigating outbreaks, including the European Centre for Disease Prevention and Control (ECDC) Outbreak Investigation Toolbox, invariably also recommend consideration of case locations [4-7]. Nevertheless, epidemiological investigations of outbreaks, and research into novel approaches for such investigations, have tended to focus more on analysis of person and time than of place [8]. Development of advanced molecular tools, for example, has allowed transmission of infectious agents among populations to be traced with ever increasing detail. Without also considering the spatial aspects of an outbreak, however, important relationships and therefore aetiological insights may be missed [8].

Geographic information systems (GIS) have increased the availability and range of tools that can be used to analyse outbreaks. A GIS is a database designed to handle geographically-referenced information complemented by software tools for the input, management, analysis and display of data [9]. GIS are used widely in epidemiology and the simplest application in an outbreak investigation is to create maps displaying the relative locations of cases, potential sources and/or risk factors. Maps are an engaging and easy-to-understand means of presenting data and can be used to describe patterns, identify outliers and communicate findings. Cases can be plotted using their point locations or aggregated into administrative areas and displayed as rates. Smoothed incidence maps are an alternative means of visualising point locations as continuous distributions of disease risk, generated by adjusting the density at each point according to the number of cases in adjacent areas [9]. Areas can also be demarcated according to locations of potential sources of infection. 


\section{FIGURE 1}

Maps of John Snow's cholera outbreak investigation in London in 1854

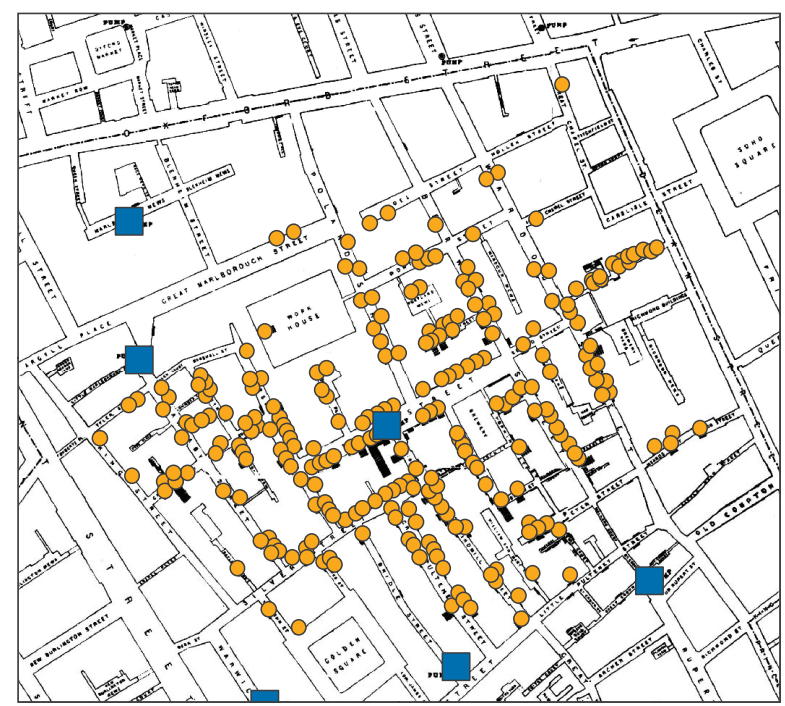

A: Dot map of case locations

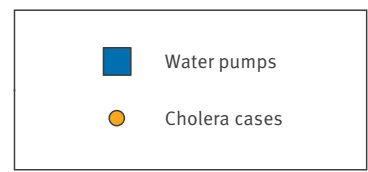

N
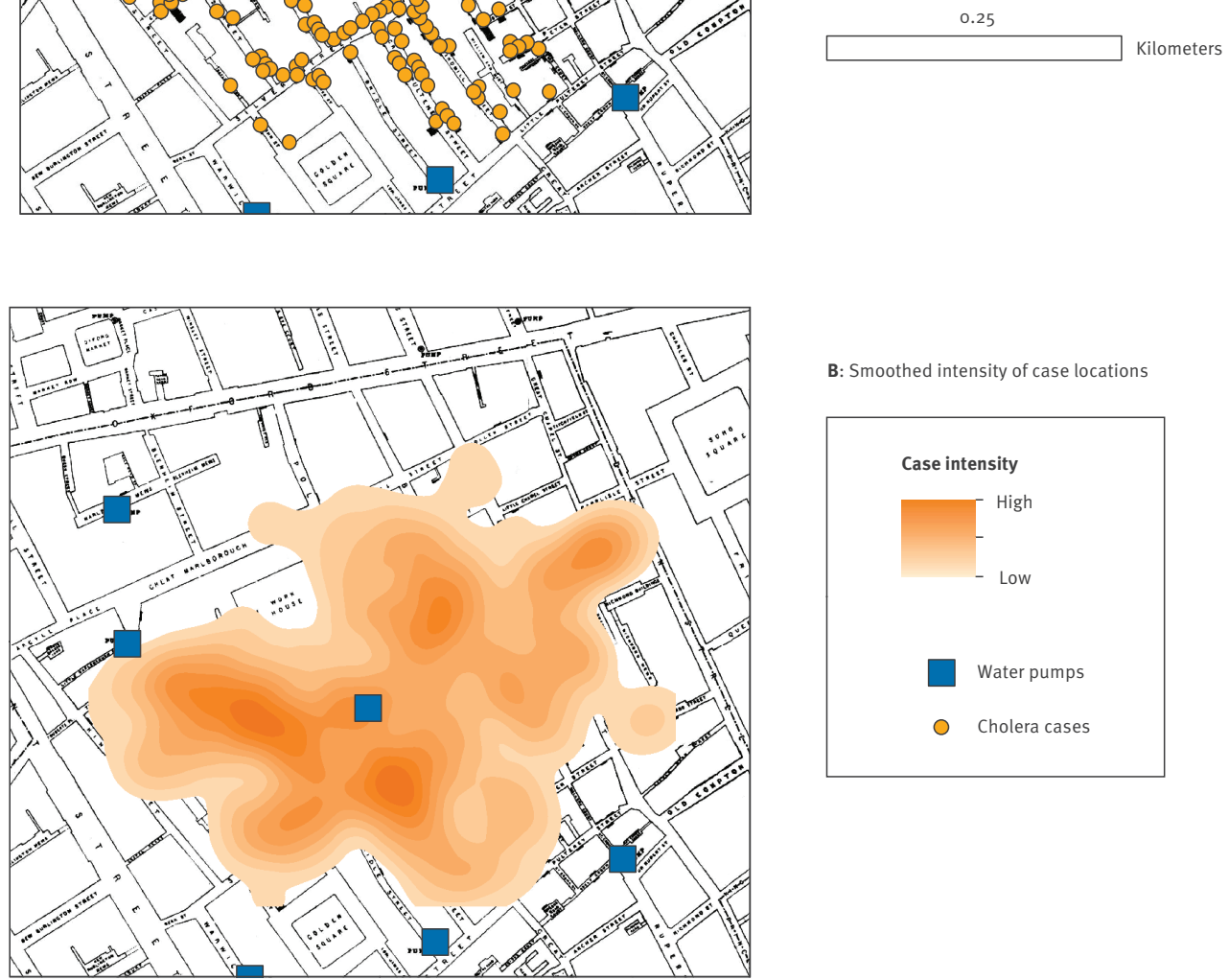

B: Smoothed intensity of case locations

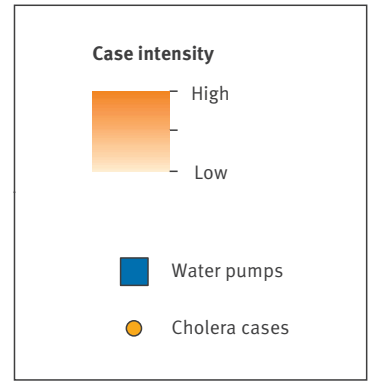

: Voronoi diagram demarcating area according to nearest water pump

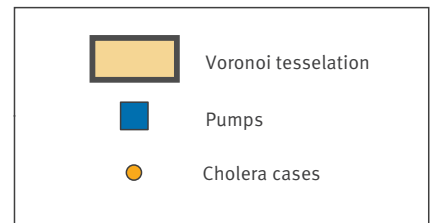

Contains Ordnance Survey data $\odot$ Crown copyright and database right 2014

and database right 2014 .

Maps produced using ArcGIS 10.2 
Examples of these different approaches to mapping, using Snow's cholera data, are shown in Figure 1.

Spatial relationships not immediately apparent from maps can also be explored using GIS. Measuring distances from cases to potential sources, for example, can be informative if an infection is suspected to derive from an environmental point source. In outbreaks of Legionnaires' disease, this method has been applied to identify cooling towers or other aerosol-producing devices proximal to the cases and therefore generate hypotheses about the likely source [10]. Integration of additional data in the GIS, such as wind direction, can further aid hypothesis generation, for example by identifying areas most likely to be exposed to air emitted from a suspected source during an outbreak of $Q$ fever [11].

Identification and analysis of clusters, areas with higher than expected levels of disease risk, can trigger and be informative during outbreak investigations. Numerous geostatistical methods have been developed to detect clusters, including methods for point and aggregated data $[9,12]$. 'Global' tests evaluate the entire area for any evidence of clustering, without pinpointing specific clusters, while 'local' (or 'cluster detection') tests identify the positions of specific clusters. Cuzick and Edwards' k-nearest neighbour test, for example, is a global method for assessing clustering in case-control point data [13]. It counts the number of nearest neighbours of cases that are also cases, and compares it to the number that would be expected under the null hypothesis that cases and controls were randomly distributed. Kulldorff's spatial scan statistic is a method used to identify local clustering, usually in point data [14]. Observed numbers of cases within windows of various sizes are compared with numbers that would be expected under a random distribution. Circular or elliptical regions of elevated risk of disease are then located. Scan statistics and the $k$-nearest neighbour test have also been adapted to identify spatiotemporal clustering, testing the null hypothesis that cases geographically close to each other occur at random times $[15,16]$.

Spatial relationships in outbreak data can also be analysed through modelling. A range of techniques can be used which, broadly, aim to create informative representations of features, events and processes in geographical space. Environmental risk mapping, for example, uses statistical methods to define relationships between spatially referenced variables and disease risk [9]. Air dispersion models, meanwhile, can be used to identify spatial locations likely to have been exposed to air-borne infections and infer potential release sites [10].

In this study, we explore through a systematic literature review how methods of spatial visualisation and analysis have been employed in infectious disease outbreak investigations. We aimed to use published reports of outbreak investigations (i) to describe the prevalence, utility and outcomes of applying spatial methods and (ii) to make recommendations for improving practice and identify opportunities for further development in this area.

\section{Methods}

Search strategy and selection criteria

The aim of our literature search was to identify published reports of infectious disease outbreak investigations that used spatial methods. We defined an outbreak as the occurrence of a series of cases of disease in excess of the number expected in a given time and place. We focused only on outbreaks with local or regional impact and excluded large national or multinational studies of epidemics or pandemics, such as pandemic influenza. Studies describing retrospective analyses of outbreaks that used spatial methods which could theoretically be applied in real-time investigations were included.

We employed a broad search strategy of multiple electronic databases with few restrictions in order to minimise the risk of bias: We searched Embase, Medline and Web of Science for items with terms relating to spatial analysis ('spatial', 'cluster', 'geographic information systems', 'GIS', 'mapping') and outbreaks ('disease outbreak', 'outbreak', 'epidemic'). The search was run on 28 November 2013 and restricted to articles published after 1980 (Embase), 1946 (Medline) and 1900 (Web of Science). No exclusions were made on basis of language or location, and articles were not limited to human disease. Additional relevant articles known to the authors that were not retrieved from the database search were also added to the results.

After deduplication, titles and abstracts were reviewed to identify articles that met our inclusion criteria: Articles had to relate to an infectious disease, they had to describe an investigation of an outbreak (as defined above) and they had to involve application of spatial analysis or mapping. Abstracts that did not include clear information on the inclusion criteria were brought forward for full-text review. Full texts of articles were assessed with the same inclusion criteria.

We then ran a search of the same databases using only the outbreak investigation terms. We simulated the deduplication and screening process that would result from this search by excluding the same proportion of articles at each step as in the original search. This allowed us to obtain a crude estimate of the total number of published reports of infectious disease outbreak investigations and therefore the proportion that used spatial methods.

\section{Data extraction}

Each included study was reviewed and information about the spatial methods and outcomes of the studies extracted (Table 1). Descriptive details obtained 


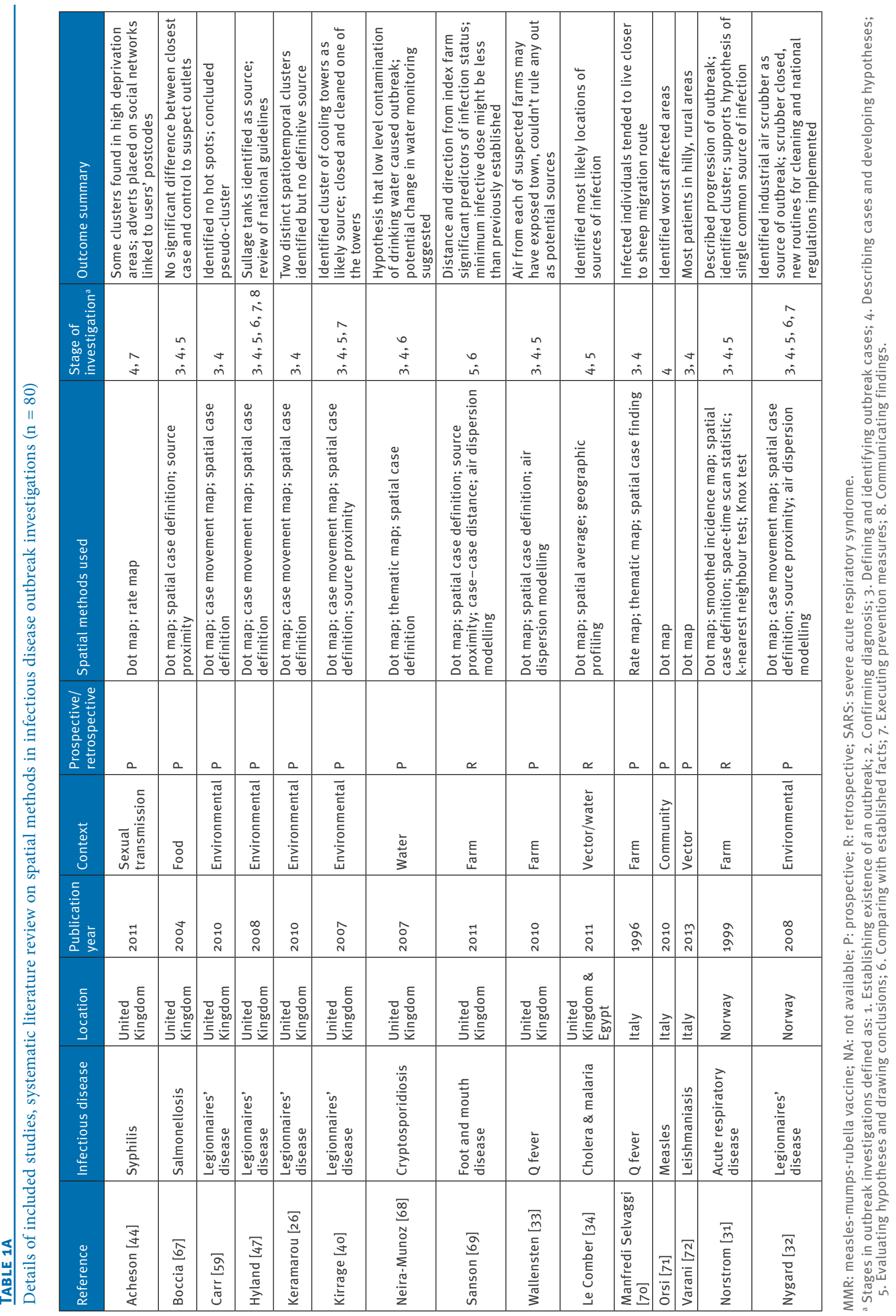




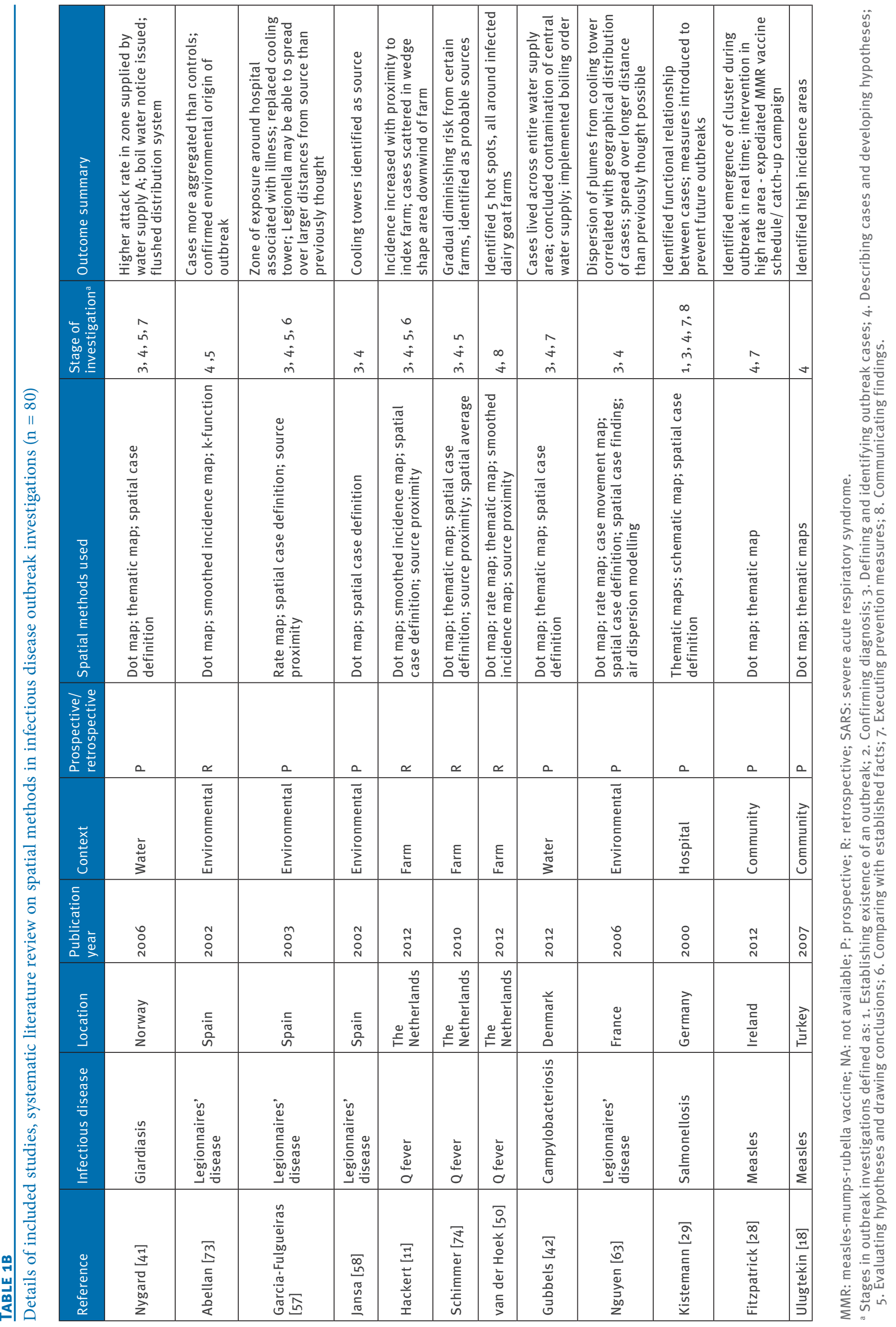




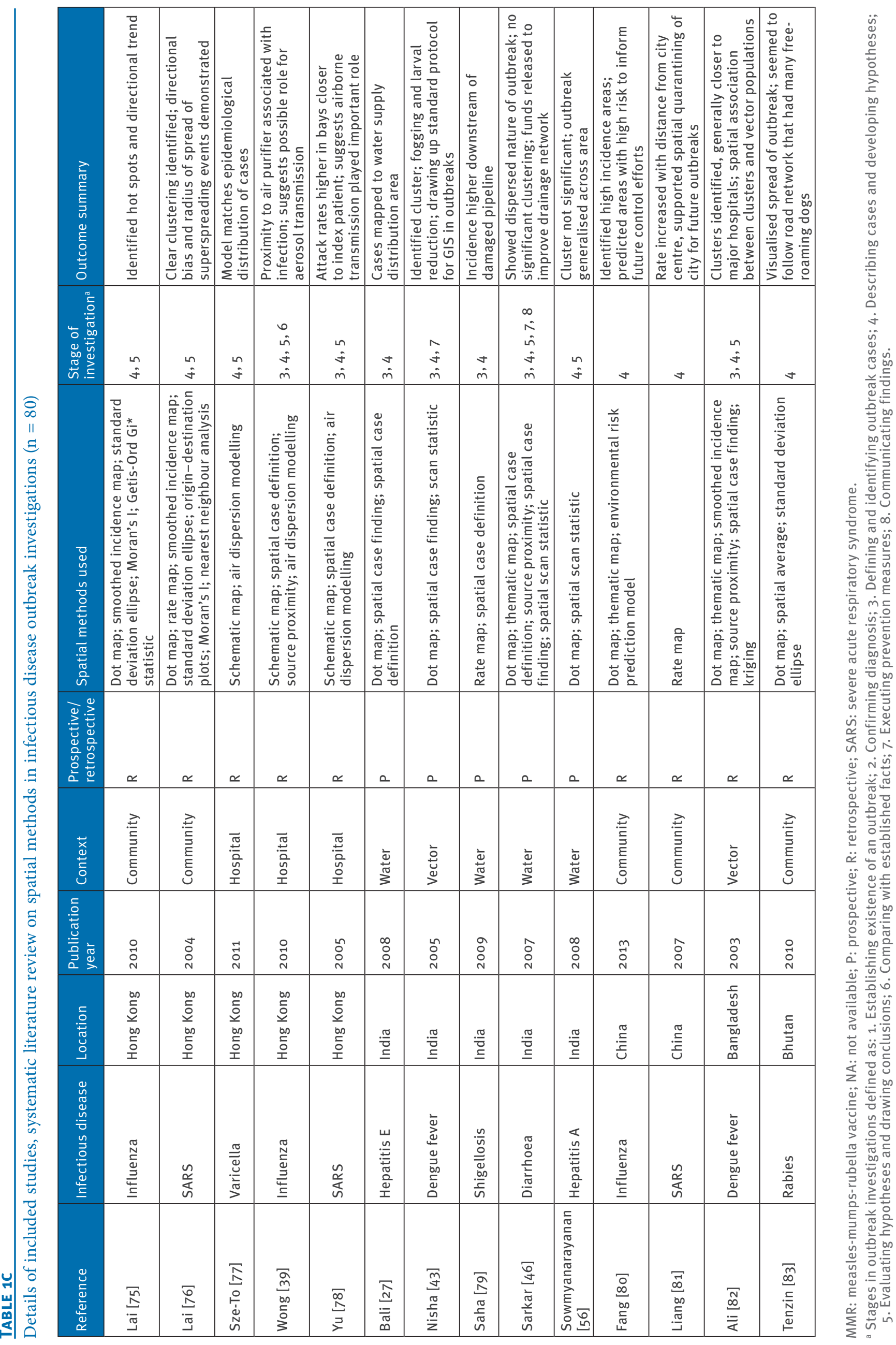




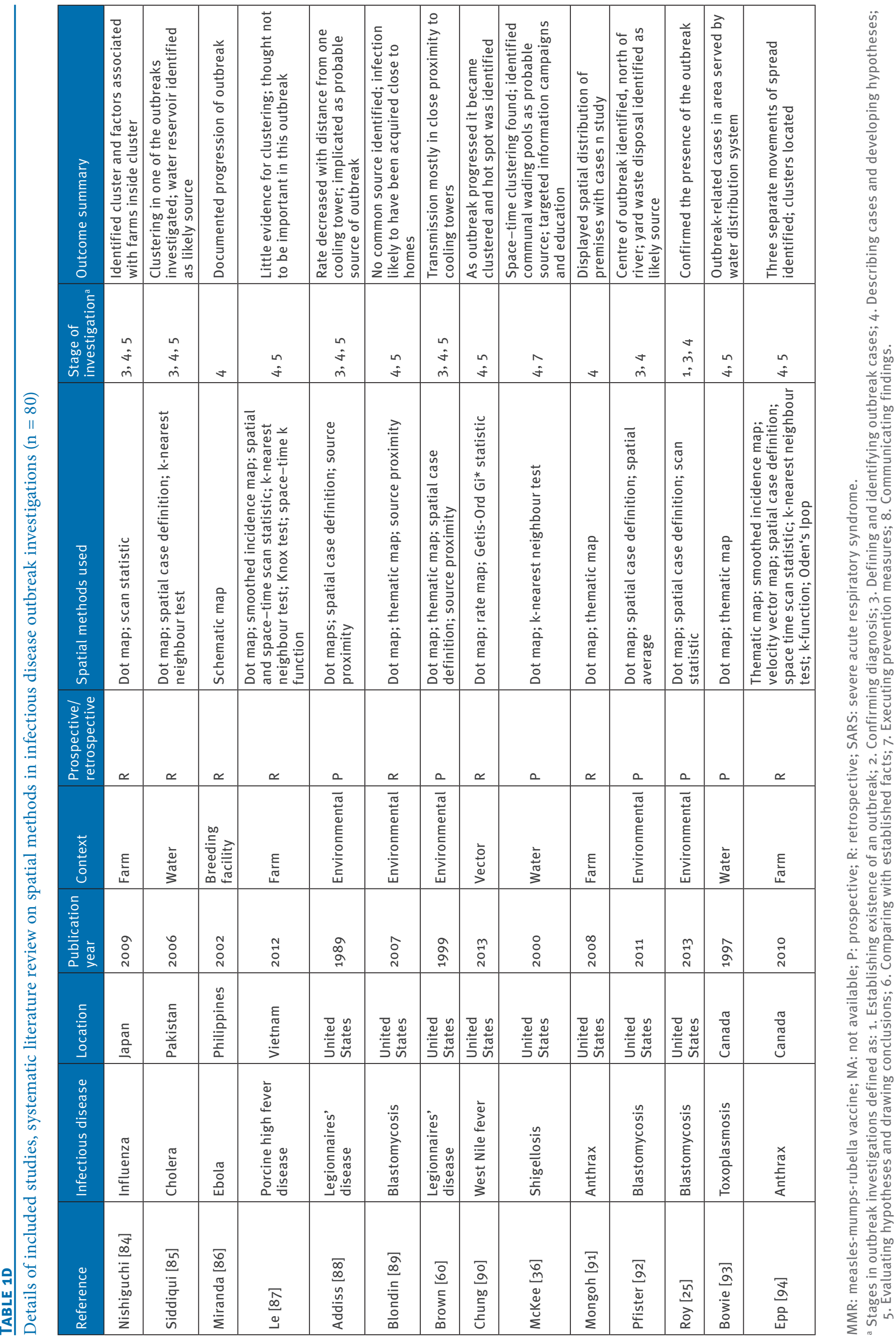




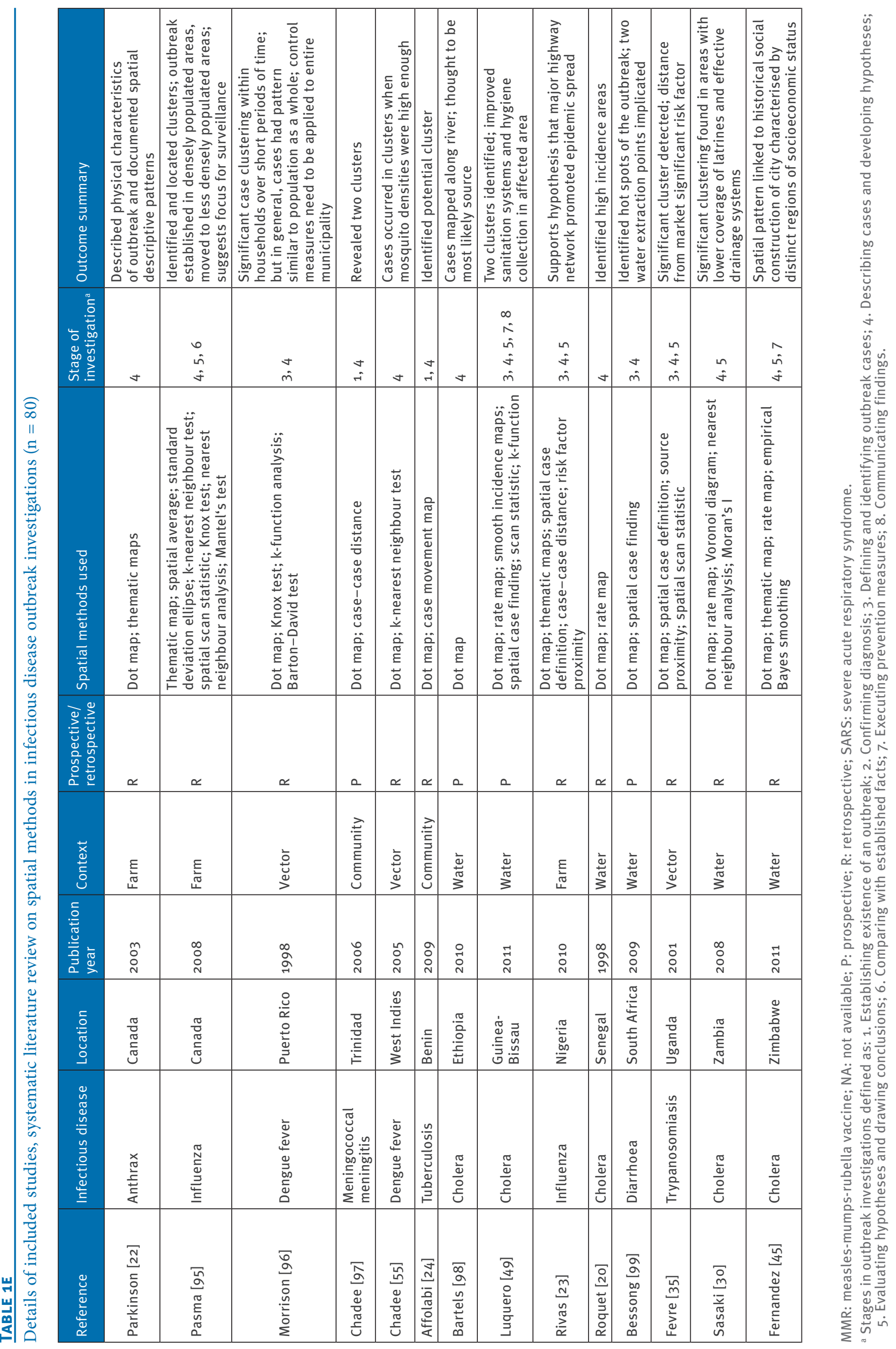




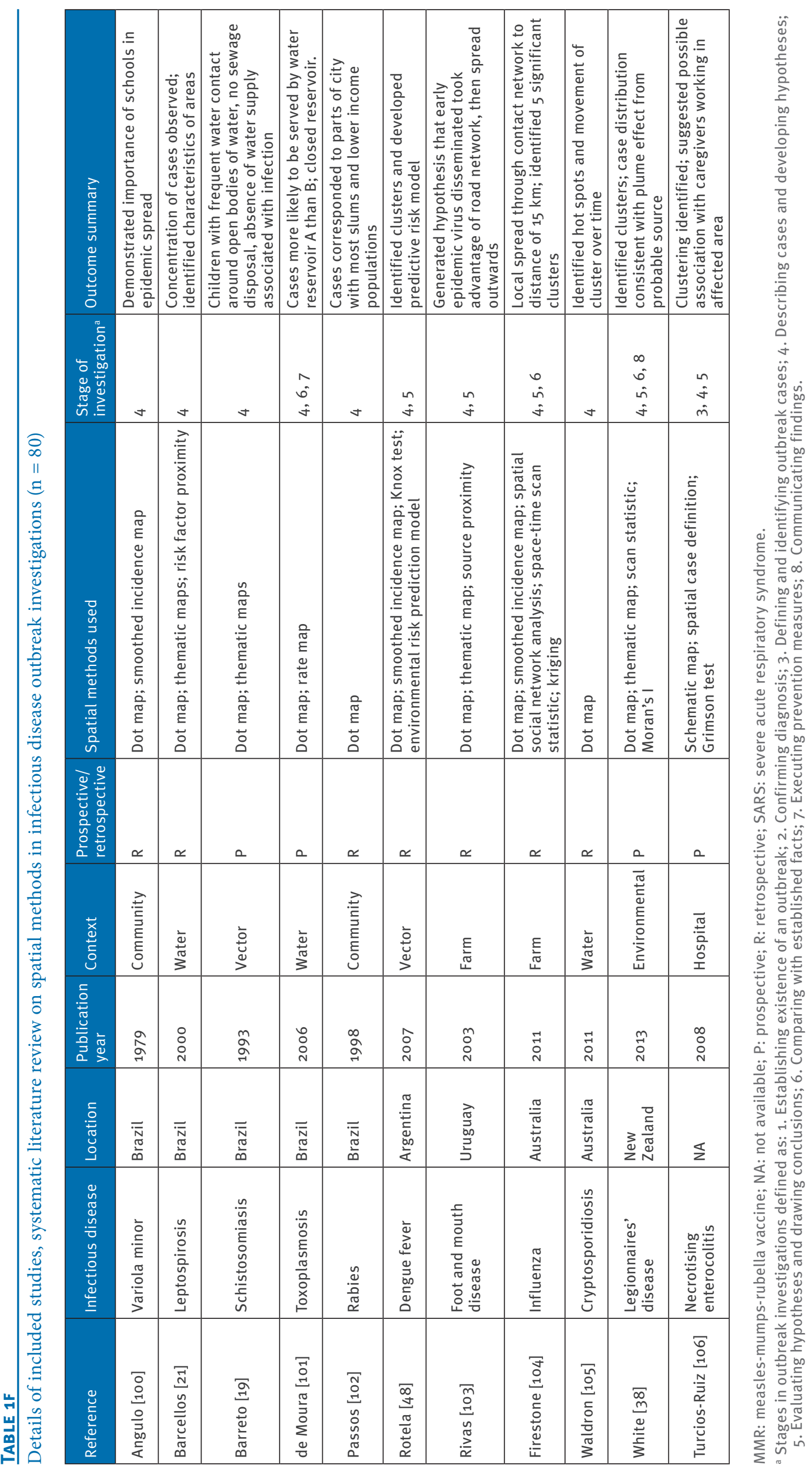




\section{FIGURE 2}

Study selection, systematic literature review on spatial methods in infectious disease outbreak investigations $(n=3,696)$

A. Literature search for outbreak investigations using spatial methods

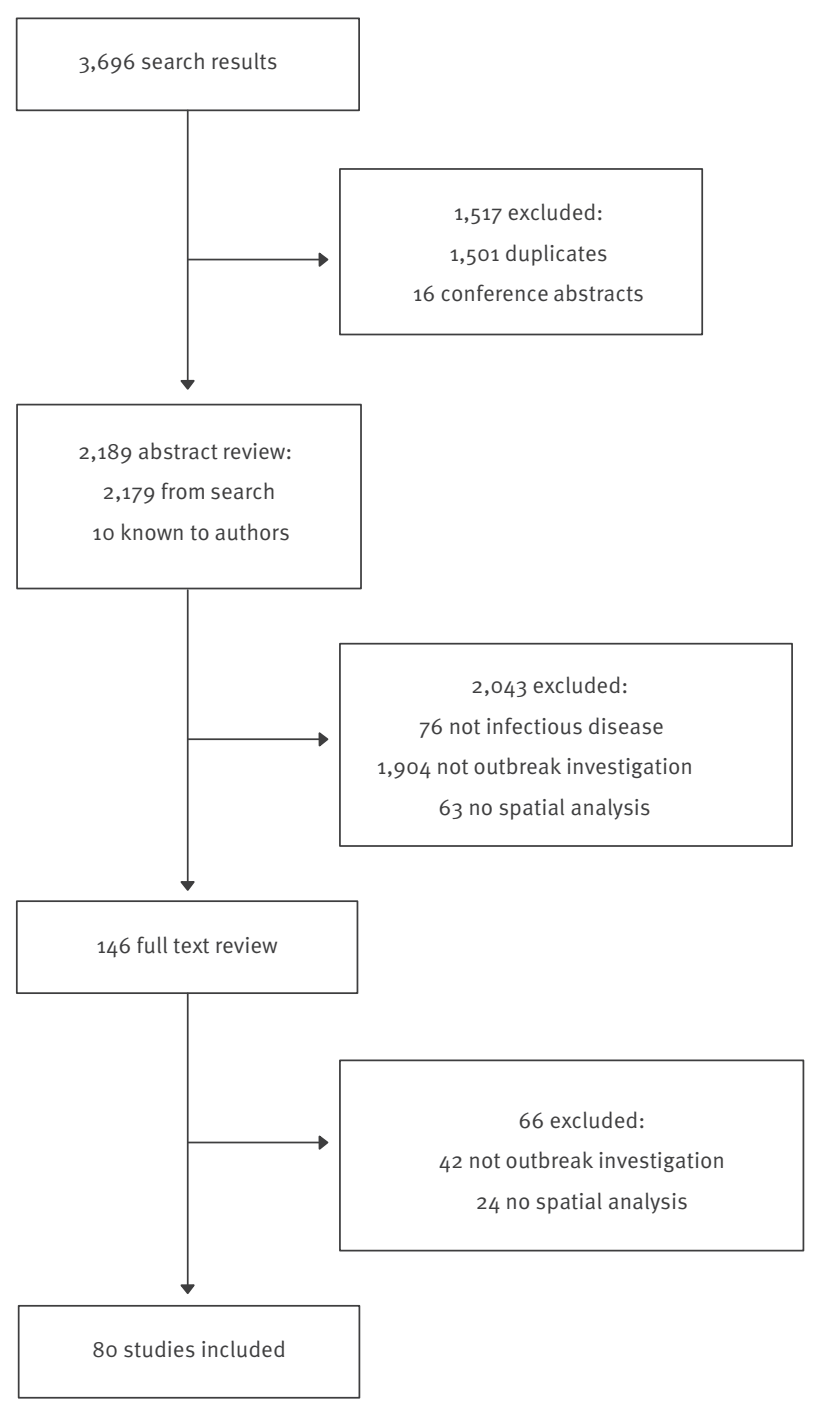

B. Simulated literature search for all outbreak investigations, using the same rate of article exclusion as in panel $A$

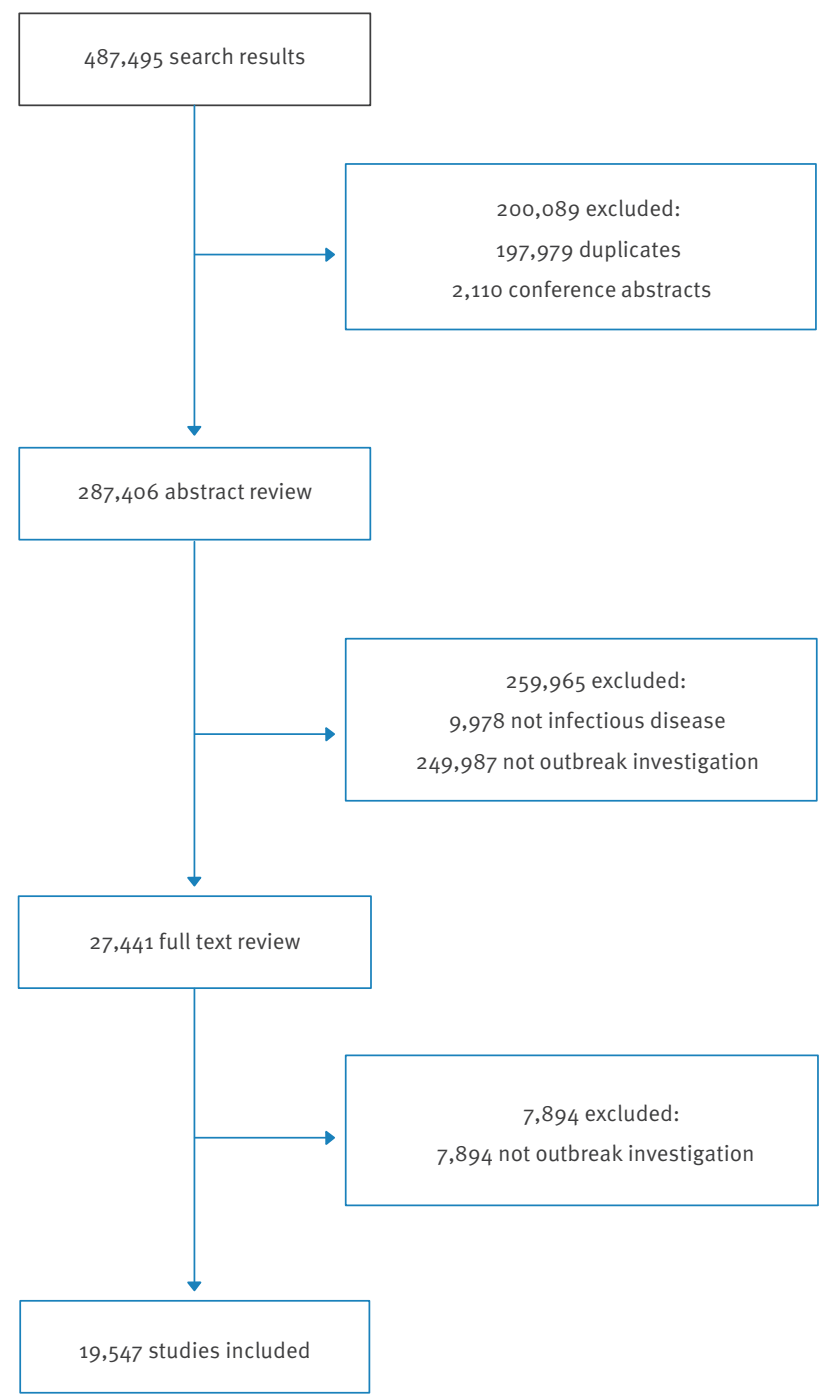

Blue boxes are estimated numbers. Key details of all 80 included articles are described in Table 1.

were the location of the outbreak, date of publication, type of infection, context or suspected source, and whether the study was prospective or retrospective. Methodological details were the type of spatial methods used and the tools employed. Outcomes were results of the investigations that related specifically to the use of spatial methods and any comments on their advantages or limitations. We summarised reports according to the date of publication, type of infection, location and context of the outbreak. Spatial methods used were categorised into four broad classes: visualisation, cluster analysis, modelling and other spatial analyses.

To demonstrate the utility of spatial methods during outbreak investigations, and therefore how they could be used in the future, we identified the stage(s) of the investigation to which they were applied. Outbreak investigations can be delineated into steps in various ways, and for the purpose of this review we used the following steps, adapted from the ECDC's Field Epidemiology Manual [17]: 1. Establishing existence of an outbreak, 2. confirming diagnosis, 3 . defining and identifying outbreak cases, 4 . describing cases and developing hypotheses, 5 . evaluating hypotheses and drawing conclusions, 6. comparing with established facts, 7. executing prevention measures, 8 . communicating findings. 
Reports of outbreak investigations using spatial methods $(n=80)$

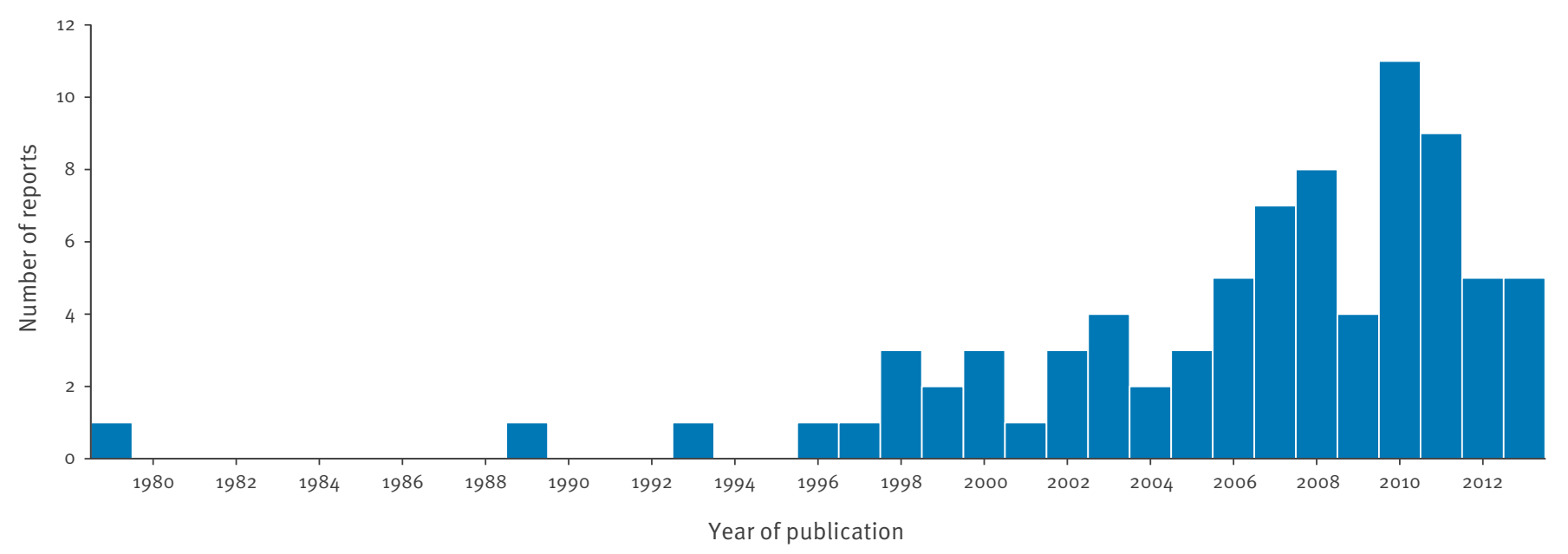

\section{Results}

Article screening and estimation of proportion with spatial methods

After excluding duplicates, we identified a total of 2,189 articles for abstract screening. Of these, 146 were selected for full text review and 80 of them were included in the analysis. Reasons for article exclusion are summarised in Figure 2A. Conducting the search without any terms specific to spatial analysis identified 487,495 articles. Assuming the same rate of article exclusion at each step in the review process, we estimated the total number of published articles relating to outbreak investigations of infectious diseases at ca 20,000 (Figure 2B). The overall proportion of published outbreak investigation reports that explicitly described spatial methods was therefore around $0.4 \%$.

\section{Characteristics of studies included}

Publication of outbreak investigations with spatial methods has increased markedly since 2000 , with over half $(n=42)$ of the studies published since 2008 (Figure 3$)$. Most articles $(n=66 ; 83 \%)$ concerned infections in human populations, of which the most frequently investigated infections were Legionnaires' disease $(n=12)$, cholera $(n=7)$ and influenza $(n=7)$ (Table 2). Correspondingly, the most common transmission contexts for human infections were water/sanitation $(n=20)$, followed by environmental $(n=14)$ and community $(n=10)$ (Table 3$)$.

Healthcare-associated infections were reported in five of the articles while food-borne and sexually transmitted infections were reported once apiece. Veterinary infections were almost exclusively linked to farms or other breeding facilities $(n=12)$ and influenza was the most frequently investigated infection affecting animals $(n=4)$. Prospective outbreak investigations comprised around half $(n=39)$ of the articles included, with the remainder describing retrospective analyses of outbreak data.

Figure 4 displays the outbreaks by country, with the most reports in the United Kingdom (UK) $(n=10)$ or the United States (US) ( $n=8)$, and by continent, with a third of reports in Europe $(n=27)$ and fewer in Africa $(n=10)$.

\section{Spatial methods}

Spatial methods used are listed and classified according to type in Table 4 .

All articles presented or referred to at least one method of visualising case distributions to describe outbreaks in space. Plotting cases as dots on a map is the simplest form of visualisation and was used in the majority $(n=68 ; 85 \%)$ of studies. Dot maps were either presented using case locations only, or were enhanced with further information such as their vaccination status [18], migratory status [19] or date of disease onset [20]. Thematic maps provide context to case locations by displaying the spatial distributions of other variables. Such maps were used in 25 studies and variables plotted included socioeconomic status [21], soil type [22] and road density [23]. Maps of disease rates were used in 14 studies, with data usually aggregated according to administrative boundaries. Smoothed incidence maps were used in 13 studies. Other methods for visualising outbreaks that were used in fewer studies included standard deviation ellipses and velocity vector maps. Both use the locations of cases to describe the direction of spread of outbreaks.

Cluster analyses were used in 24 studies (30\%), and spatial scan statistics were the most frequently used ( $\mathrm{n}=13$ studies). $k$-nearest neighbour tests, $k$-function analyses and the Knox test were also used frequently ( $n=7,5$ and 5 studies, respectively). Modelling approaches were used in 13 studies, including seven which used air dispersion models to identify areas that 
TABLE 2

Infectious diseases investigated by category $(n=80$ reports)

\begin{tabular}{|c|c|c|c|c|}
\hline $\begin{array}{l}\text { Infection } \\
\text { category }\end{array}$ & $\mathrm{n}$ & Infection & $\mathrm{n}^{\mathrm{a}}$ & References \\
\hline \multirow{4}{*}{ Respiratory } & \multirow{4}{*}{23} & $\begin{array}{l}\text { Legionnaires' } \\
\text { disease }\end{array}$ & 12 & $\begin{array}{l}{[26,32,38,40,47,57-} \\
60,63,73,88]\end{array}$ \\
\hline & & Influenza & 7 & {$[23,39,75,80,84,95,104]$} \\
\hline & & SARS & 3 & {$[76,78,81]$} \\
\hline & & $\begin{array}{l}\text { Acute respiratory } \\
\text { disease }\end{array}$ & 1 & [31] \\
\hline \multirow{8}{*}{ Intestinal } & \multirow{8}{*}{18} & Cholera & 7 & {$[20,30,34,45,49,85,98]$} \\
\hline & & Cryptosporidiosis & 2 & {$[68,105]$} \\
\hline & & Diarrhoea & 2 & {$[46,99]$} \\
\hline & & Salmonellosis & 2 & {$[29,67]$} \\
\hline & & Shigellosis & 2 & {$[36,79]$} \\
\hline & & Campylobacteriosis & 1 & [42] \\
\hline & & Giardiasis & 1 & [41] \\
\hline & & $\begin{array}{l}\text { Necrotising } \\
\text { enterocolitis }\end{array}$ & 1 & [106] \\
\hline \multirow{4}{*}{$\begin{array}{l}\text { Viral } \\
\text { haemorrhagic } \\
\text { fever }\end{array}$} & \multirow{4}{*}{8} & Dengue fever & 5 & {$[43,48,55,82,96]$} \\
\hline & & Ebola & 1 & {$[86]$} \\
\hline & & $\begin{array}{l}\text { Porcine high fever } \\
\text { disease }\end{array}$ & 1 & [87] \\
\hline & & West Nile fever & 1 & [9o] \\
\hline \multirow{4}{*}{$\begin{array}{l}\text { Viral skin } \\
\text { infections }\end{array}$} & \multirow{4}{*}{7} & Measles & 3 & {$[18,28,71]$} \\
\hline & & $\begin{array}{l}\text { Foot and mouth } \\
\text { disease }\end{array}$ & 2 & {$[69,103]$} \\
\hline & & Varicella & 1 & {$[77]$} \\
\hline & & Variola minor & 1 & [100] \\
\hline \multirow{4}{*}{ Protozoal } & \multirow{4}{*}{5} & Toxoplasmosis & 2 & {$[93,101]$} \\
\hline & & Leishmaniasis & 1 & {$[72]$} \\
\hline & & Malaria & 1 & [34] \\
\hline & & Trypanosomiasis & 1 & [35] \\
\hline Rickettsioses & 5 & Q fever & 5 & {$[11,33,50,70,74]$} \\
\hline \multirow{2}{*}{$\begin{array}{l}\text { Bacterial } \\
\text { zoonotic }\end{array}$} & \multirow{2}{*}{4} & Anthrax & 3 & {$[22,91,94]$} \\
\hline & & Leptospirosis & 1 & {$[21]$} \\
\hline Mycoses & 3 & Blastomycosis & 3 & {$[25,89,92]$} \\
\hline $\begin{array}{l}\text { Viral CNS } \\
\text { infections }\end{array}$ & 2 & Rabies & 2 & {$[83,102]$} \\
\hline \multirow{2}{*}{ Viral hepatitis } & \multirow{2}{*}{2} & Hepatitis A & 1 & [56] \\
\hline & & Hepatitis E & 1 & {$[27]$} \\
\hline Helminthiases & 1 & Schistosomiasis & 1 & [19] \\
\hline $\begin{array}{l}\text { Other } \\
\text { bacterial }\end{array}$ & 1 & $\begin{array}{l}\text { Meningococcal } \\
\text { meningitis } \\
\end{array}$ & 1 & [97] \\
\hline $\begin{array}{l}\text { Sexually } \\
\text { transmitted }\end{array}$ & 1 & Syphilis & 1 & [44] \\
\hline Tuberculosis & 1 & Tuberculosis & 1 & [24] \\
\hline
\end{tabular}

CNS: central nervous system; SARS: severe acute respiratory syndrome.

a The total is 81 because one study reported two investigations.

may have been exposed to air from suspected contaminated environmental sources.

A range of other spatial methods based on geographic attributes of cases were also identified. These included methods for defining ( $n=31$ studies) and identifying ( $n=8$ studies) cases, summarising the average locations of cases ( $n=5$ studies) and assessing proximity to potential sources ( $n=16$ studies).

Analytic methods were used less frequently in prospective than retrospective articles: Cluster methods were used in 16 (39\%) retrospective compared with eight (21\%) prospective studies, and modelling in 10 (24\%) and three ( $8 \%$ ) retrospective and prospective analyses, respectively.

The most frequently cited GIS software was ArcGIS/ ArcView, used in 30 studies, with Maplnfo the other commonly used programme $(n=7)$. Various other packages including R, ClusterSeer, GeoDa and SaTScan were used for specific analyses.

\section{Application of spatial methods in outbreak investigations}

Applications of spatial methods to different stages during outbreak investigations are described below (see also Table 1).

\section{Establishing existence of an outbreak}

Few studies $(n=4)$ used spatial methods to assist with establishing the existence of an outbreak. Methods that were used aimed to identify unusual patterns of cases, either visually or through formal statistical tests of clustering.

For example, Affolabi and colleagues described complementary use of molecular and geographic methods to identify an outbreak of tuberculosis in Benin [24]. Among a series of $194 \mathrm{M}$. tuberculosis isolates, 17 belonged to the Beijing genotype and exhibited an identical 12-loci subtype. Mapping of patients' residences, workplaces and movements revealed a corresponding spatial cluster, confirming that the cases were likely to be linked. In another study, Roy and colleagues plotted the locations of cases of blastomycosis in Wisconsin after noting an increase in the number of reports [25]. They visually identified clustering within five neighbourhoods and used the spatiotemporal scan statistic to confirm that this was statistically significant.

\section{Confirming diagnosis}

Although knowledge of the endemicity of diseases in the geographic regions in which outbreaks arise is useful in developing plausible preliminary diagnostic hypotheses, spatial methods alone are not able to confirm a diagnosis and were therefore not used for this purpose in any of the studies.

\section{Defining and identifying outbreak cases}

Geographic boundaries in which outbreak cases were defined were stated explicitly in over a third $(n=31)$ of the studies. For instance, Keramarou and colleagues' investigation of an outbreak of Legionnaires' disease included only cases that lived or worked in the outbreak area, defined as a $12 \mathrm{~km}$ corridor on either side of a major road [26]. 
TABLE 3

Contexts of outbreak investigations of human and animal diseases ( $\mathrm{n}=80$ reports)

\begin{tabular}{|c|c|c|c|c|}
\hline \multirow{2}{*}{ Context } & \multicolumn{3}{|c|}{ Humana $^{a}$} & \multirow{2}{*}{$\begin{array}{c}\text { Animal } \\
\text { References }\end{array}$} \\
\hline & $\mathrm{n}$ & References & $\mathrm{n}$ & \\
\hline Water/sanitation & 20 & $\begin{array}{c}{[20,21,27,30,34,36,41,42,45,46,49,56,68,79,85} \\
93,98,99,101,105]\end{array}$ & 0 & \\
\hline Environmental & 14 & {$[25,26,32,38,40,47,57-60,63,73,88,92]$} & 1 & [89] \\
\hline Community & 10 & {$[18,24,28,71,75,76,80,81,97,100]$} & 2 & {$[83,102]$} \\
\hline Vector-borne & 10 & {$[19,34,35,43,48,55,72,82,90,96]$} & 0 & \\
\hline Farm/breeding facility & 5 & {$[11,33,50,70,74]$} & 12 & $\begin{array}{c}{[22,23,31,69,84,86,87} \\
91,94,95,103,104]\end{array}$ \\
\hline Healthcare-associated & 5 & {$[29,39,77,78,106]$} & 0 & \\
\hline Food & 1 & [67] & 0 & \\
\hline Sexually transmitted & 1 & [44] & 0 & \\
\hline Total b & 66 & & 15 & \\
\hline
\end{tabular}

a Includes outbreaks affecting humans that had animal origin.

b The total is 81 because one article reported two investigations.

Spatial methods were also used to assist with active case finding in eight studies. Bali and colleagues describe a search for cases of hepatitis E prompted by identification of three cases in a small town in northern India [27]. A house-to-house survey in this region identified 3,170 cases of jaundice with an attack rate of $5.2 \%$.

4. Describing outbreak cases and developing hypotheses Use of dot mapping to support an outbreak in real time is described by Fitzpatrick and colleagues, who investigated a rise in measles cases in Dublin, Ireland [28]. Continuously updating their maps throughout the outbreak allowed them to identify clustering of cases as soon as it developed and ultimately assisted with targeting of control interventions.

Simple maps were also used to develop hypotheses about the origins of outbreaks. For example, Kistemann and colleagues plotted cases by date of onset in an investigation of a nosocomial Salmonella outbreak [29]. Their schematic map revealed the central kitchen as the only functional relationship linking the cases, which they therefore hypothesised to be the source of the infection.

Sasaki and colleagues created a Voronoi diagram to demarcate their study area using locations of water taps [30]. Plotting incidence rates in the different areas defined by these water tap boundaries helped to visualise clear spatial clustering of cholera cases associated with poor water and sanitation facilities. Smoothed incidence maps were used in an investigation by Norström and colleagues into acute respiratory disease in Norwegian cattle herds. They used smoothing based on kernel density estimation to describe the progression of the outbreak, which was shown to spread locally before jumping to new areas [31].
A common method to develop hypotheses about sources of infections was to construct concentric circles of varying radii around potential sources and compare the attack rates in each. Nygard and colleagues used this technique in an investigation of Legionnaires' disease in Norway [32]. They calculated attack rates in five rings of increasing distance around eight potential sources and observed a trend of decreasing rate ratios with increasing distance from an air scrubber. Other metrics used to describe cases included calculating their average location and proximity to risk factors.

Possible air-borne spread of $Q$ fever from farms near Cheltenham, UK was investigated by Wallensten and colleagues using the Numerical Atmosphericdispersion Modelling Environment (NAME) model [33]. Plotting the modelled distribution showed that air from each of the suspected farms may have exposed the town. Geographic profiling is another modelling technique used to generate hypotheses about the locations of sources of infections. Le Comber and colleagues used this method to identify most likely locations of mosquito breeding sites using residential locations of a series of cases of malaria in Cairo, Egypt [34].

5. Evaluating hypotheses and drawing conclusions More than half of the studies $(n=42)$ used statistical tests, such as cluster and regression analyses, to conduct formal evaluations of hypotheses arising from observations of case distributions. Fevre and colleagues, for example, assessed clustering of cases of trypanosomiasis under the hypothesis that a cattle market was the source of the outbreak [35]. A significant cluster encompassing the location of the market was detected using the spatiotemporal scan statistic, supporting this theory.

In an investigation on a military installation in North Carolina, McKee and colleagues used the $k$-nearest 


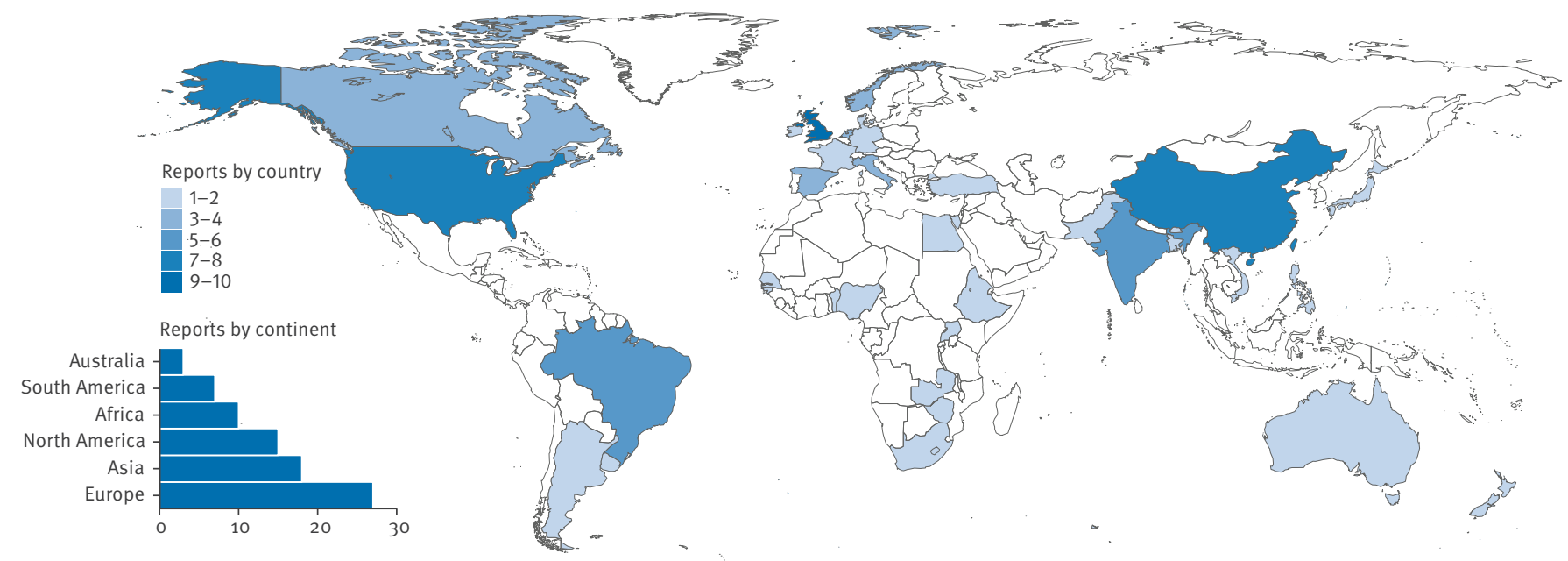

neighbour method to identify significant spatiotemporal clustering of shigellosis [36]. They used dot maps to locate the area with intense transmission and targeted it with educational interventions to bring the outbreak under control.

Combinations of multiple tests for clustering were used in some studies, such as Norström and colleagues' investigation of acute respiratory disease in Norwegian cattle herds [31]. They combined the Knox test [37], a global test for space-time clustering, with the $k$-nearest neighbour test and space-time scan statistic. These tests allowed them, respectively, to define the smallest distance and time frame in which the events had been clustered, to determine whether cases tended to be close to other cases and to locate the most significant clusters. All methods indicated presence of space-time clustering, adding weight to the conclusion that a common contagious source was responsible for the outbreak.

The hypothesis that risk of infection decreased with increasing distance from a suspected source was tested in several studies through regression analysis. Hackert and colleagues, for example, used linear regression of log-transformed attack rates to assess a cluster of human cases of $Q$ fever in the Netherlands [11]. Incidence increased by a statistically significant exposure-response gradient with proximity to a dairy goat farm, which they concluded was likely to be the primary and sole source.

\section{Comparing results with established facts}

Results from spatial analyses in some cases provided updates to knowledge about the dynamics of the infectious agents concerned, such as their minimum infective dose and mode of transmission. For example, in an outbreak of Legionnaires' disease in Christchurch, New Zealand, cases were identified at a distance of 12 $\mathrm{km}$ from the implicated cooling tower [38]. White and colleagues therefore proposed updates to World Health Organization guidelines which at the time placed the area at risk from such sources at $3.2 \mathrm{~km}$.

Wong and colleagues used a computational fluid dynamics model to study the spread of an influenza outbreak in a hospital setting [39]. Concentrations of hypothetical virus-laden particles from modelled air distributions correlated closely with locations of infected patients. This suggested a possible role for aerosol transmission of influenza, which is predominantly associated with transmission by droplets and direct contact.

\section{Executing prevention measures}

Spatially targeted interventions to control the outbreak or prevent future cases were described in many studies. Measures that aimed to control outbreaks included cleaning implicated cooling towers [40], issuing water boiling orders to areas served by contaminated supplies [41,42], vaccination catch-up campaigns [28], removal of breeding sites for mosquito larvae [43] and targeted information campaigns [36]. For example, Acheson and colleagues placed postcode-targeted information on social networks during an outbreak of heterosexually acquired syphilis in Teesside, UK [44].

Attempts to prevent future outbreaks included improvement of infrastructure $[45,46]$, change of policy $[29,32,47]$ and generation of risk maps [48]. Luquero and colleagues, for instance, used results of their analysis to recommend specific regions in which to focus preparedness activities to avoid future cholera outbreaks in Guinea-Bissau [49].

\section{Communicating findings}

All studies in this review had, by definition, used their spatial analyses in communication of findings through reports in peer-reviewed publications. Several studies also highlighted the usefulness of maps in reports or 
TABLE 4

Spatial methods used in outbreak investigations $(n=80)$

\begin{tabular}{|c|c|c|c|c|}
\hline Method category & $\begin{array}{l}n \text { (prospective, } \\
\text { retrospective) }\end{array}$ & Method & $n$ & References \\
\hline \multirow{10}{*}{ Visualisation } & \multirow{10}{*}{$80(39,41)$} & Dot map & 68 & $\begin{array}{l}{[11,18-28,30-36,38,40-50,55,56,58-60} \\
63,67-69,71-76,80,82-85,87-93,96-105]\end{array}$ \\
\hline & & Thematic map & 25 & $\begin{array}{l}{[18,19,21-23,28,29,38,41,42,45,46,50,} \\
60,68,70,74,80,82,89,91,93-95,103] \\
\end{array}$ \\
\hline & & Rate map & 14 & $\begin{array}{l}{[20,30,44,45,49,50,57,63,70,76,79,81,} \\
90,101]\end{array}$ \\
\hline & & Smoothed incidence map & 13 & {$[11,31,48-50,73,75,76,82,87,94,100,104]$} \\
\hline & & Case movement map & 7 & {$[24,26,32,40,47,59,63]$} \\
\hline & & Schematic map & 6 & {$[29,39,77,78,86,106]$} \\
\hline & & Standard deviation ellipse & 4 & {$[75,76,83,95]$} \\
\hline & & Origin-destination plot & 1 & [76] \\
\hline & & Velocity vector map & 1 & [94] \\
\hline & & Voronoi diagram & 1 & [30] \\
\hline \multirow{7}{*}{ Spatial exploration } & \multirow{7}{*}{$47(28,19)$} & Spatial case definition & 32 & $\begin{array}{l}{[11,23,25-27,29,31-33,35,39-42,46,47,} \\
57-60,63,67-69,74,78,79,85,88,92,94,106]\end{array}$ \\
\hline & & Source proximity & 16 & $\begin{array}{l}{[11,32,35,39,40,46,50,57,60,67,69,74,} \\
82,88,89,103]\end{array}$ \\
\hline & & Spatial case finding & 8 & {$[27,43,46,49,63,70,82,99]$} \\
\hline & & Spatial average & 5 & {$[34,74,83,92,95]$} \\
\hline & & Case-case distance & 3 & {$[23,69,97]$} \\
\hline & & Risk factor proximity & 2 & {$[21,23]$} \\
\hline & & Spatial social network analysis & 1 & {$[104]$} \\
\hline \multirow{11}{*}{ Cluster } & \multirow{11}{*}{$24(8,16)$} & $\begin{array}{l}\text { Kulldorff's spatial/ spatiotemporal scan } \\
\text { statistic }\end{array}$ & 13 & {$[25,31,35,38,43,46,49,56,84,87,94,95,104]$} \\
\hline & & $\begin{array}{l}\text { Cuzick-Edwards } k \text {-nearest neighbour } \\
\text { test/Jacquez's } k \text {-nearest neighbours for } \\
\text { space time interaction }\end{array}$ & 7 & {$[31,36,55,85,87,94,95]$} \\
\hline & & Knox test & 5 & {$[31,48,87,95,96]$} \\
\hline & & $k$-function/space-time $k$-function & 5 & {$[49,73,87,94,96]$} \\
\hline & & Moran's I & 4 & {$[30,38,75,76]$} \\
\hline & & Nearest neighbour analysis & 3 & {$[30,76,95]$} \\
\hline & & Getis Ord Gi(d) statistic & 2 & {$[75,90]$} \\
\hline & & Barton-David's test & 1 & [96] \\
\hline & & Grimson test & 1 & {$[106]$} \\
\hline & & Oden's Ipop & 1 & [94] \\
\hline & & Mantel's test & 1 & [95] \\
\hline \multirow{5}{*}{ Spatial modelling } & \multirow{5}{*}{$13(3,10)$} & Air dispersion modelling & 7 & {$[32,33,39,63,69,77,78]$} \\
\hline & & Environmental risk prediction model & 2 & {$[48,80]$} \\
\hline & & Kriging & 2 & {$[82,104]$} \\
\hline & & Empirical Bayes smoothing & 1 & [45] \\
\hline & & Geographic profiling & 1 & [34] \\
\hline
\end{tabular}

presentations to communicate results to health officials $[29,47]$, policymakers [49,50] and the public [38]. Sarkar and colleagues, for example, presented dot maps of cases of acute diarrhoeal disease in a village in southern India to the local community and health authorities [46]. Their maps visualised the proximity of cases to a contaminated water supply, and the presentation resulted in release of funds to improve sanitation in the area.

\section{Discussion}

In this review, we have identified 80 published articles of infectious disease outbreak investigations that used spatial methods, less than half a per cent of our estimated total of 20,000 outbreak reports. Although the simple dot map was the most commonly used method, a wide range of techniques were applied, including more sophisticated data visualisations and analytic tools. Across the range of studies, there were examples of spatial tools being usefully applied throughout the course of an outbreak investigation; from initial confirmation of the outbreak to describing and analysing 
TABLE 5

Application of spatial methods to steps in outbreak investigation

\begin{tabular}{|l|l|}
\hline 1. Establish the existence of an outbreak & $\begin{array}{l}\text { - Visualise case distribution (e.g. dot map) } \\
\text { - Identify and confirm clustering (e.g. spatial scan statistic) }\end{array}$ \\
\hline 2. Confirm diagnosis & $\begin{array}{l}\text { - Spatial methods alone cannot confirm diagnoses. Consider spatial epidemiology of infection to } \\
\text { develop preliminary diagnostic hypotheses. }\end{array}$ \\
\hline 3. Define and identify outbreak cases & $\begin{array}{l}\text { - Set geographic limits in which cases are considered part of the outbreak (e.g. postcode area } \\
\text { hospital ward) } \\
\text { - Select controls in case-control study based on same geographic limits }\end{array}$ \\
\hline $\begin{array}{l}\text { 4. Describe cases and develop } \\
\text { hypotheses }\end{array}$ & $\begin{array}{l}\text { - Visualise distribution of cases in relation to known risk factors or potential sources (e.g. rate } \\
\text { map, thematic maps) } \\
\text { Describe progression of outbreak (e.g. dot maps at different stages, standard deviation ellipse) } \\
\text { Identify centre of outbreak (e.g. spatial mean) } \\
\text { - Identify high-risk areas (e.g. attack rates in zones at different distances from potential sources) }\end{array}$ \\
\hline 5. Evaluate hypotheses and draw \\
conclusions
\end{tabular}

cases and communicating findings. Spatial techniques often provided valuable insights that supplemented traditional epidemiological analyses of person and time and led to public health actions.

Outbreak investigations of infectious diseases occurring in any context were included in this study. Thus, we extended the scope of two previous reviews that focused, respectively, on use of spatial methods in outbreaks of Legionnaires' disease [10] and on spatiotemporal methods to investigate transmission of infections in hospital settings [51]. In doing so, we have highlighted imbalances in application of spatial methods in different types of investigations. For example, it was notable that only one study reported an outbreak of food-borne illness. Annual summary statistics from 2013 report a total of 5,196 food- and waterborne outbreaks in the European Union (EU) [52] and 831 reports of food-borne outbreaks in 2012 in the US [53]. Although only a small proportion of these are likely to have been published in academic journals, this still indicates a substantial shortfall in use of spatial methods in this context.

Our review also allowed assessment of the extent to which spatial methods have been used in Europe. Although there was a large number of reports from Europe compared with other parts of the world, those reports derived from only 10 counties. These were predominantly in western Europe, with one report from Turkey the only investigation in eastern areas. Sharing expertise through the European Centre of Disease
Prevention and Control could help to reduce this gap and strengthen outbreak investigation capacity across the continent. Expanding the use of these tools is also important in other parts of the world. Only 10 reports described outbreaks in Africa, the same number as in the UK alone, which clearly does not correlate with the distribution of the global burden of infectious diseases.

There are several limitations of spatial methods, and barriers to their use, which may account for the unequal and under-use of these tools as identified here. Firstly, reliable spatial analyses can only be conducted with accurate location data. This can be a particular challenge in developing countries in which good quality maps of residential areas are often not available [54]. Several investigations of outbreaks in such settings conducted field surveys and used Global Positioning Systems (GPS) to accurately record patient residence or risk factor locations $[30,35,43,46,55,56]$. However, this is a time- and cost-intensive approach and will not always be feasible. In settings in which good quality maps of residential data are available, quality of case location data is still not assured: Errors can arise from incomplete or mistranscribed addresses, out of date GIS databases or incomplete information on potential source locations. During outbreaks of Legionnaires' disease, for example, some investigators had to conduct visual searches or make public enquiries to ascertain the locations of aerosol-producing devices because there was no central registry $[26,32,40,57-60]$. 
Simplification of case locations to static points, usually residential locations, also impacts the utility of location data. In reality, individuals can become exposed to infectious agents at any place where they spend time and, similarly, traditional census population denominators that record night-time populations are not necessarily reflective of population distributions during the day $[61,62]$. Although a number of studies made attempts to record case movements $[24,26,32,40,47,59,63]$, none accounted for diurnal fluctuations in populations. Ideally, this spatial uncertainty should be accounted for in data collection, analysis and visualisation stages to improve reliability of estimates of spatial risk, and new analytic methods may be required to achieve this.

Secondly, even if reliable location data are available, presentation of information on maps can be open to misinterpretation. Dot maps, for instance, were used widely but do not take into account the geographic distribution of the underlying population and can therefore mask important trends. Similarly, patterns in aggregated data are sensitive to changes in the boundaries into which they are grouped, a phenomenon known as the modifiable aerial unit problem [9]. Presentation of data on maps fails to highlight these limitations, and relatively few prospective investigations used statistical methods to formally confirm observations identified from visual displays of data.

Thirdly, researchers may be deterred from using spatial analytic methods because they involve selection of parameter values, often with an element of subjectivity. Methods that display or identify clustering require specification of the degree to which distant points may be considered part of the same neighbourhood. For the spatial scan statistic, the user must define the maximum spatial extent of clusters in terms of the percentage of the population that can be included, in $k$-nearest neighbour analysis, the number of neighbours included must be specified, and equivalent parameters must be selected for other spatial cluster and modelling analyses [9]. Altering these parameters can have a profound influence on the results, and a trial and error approach is often required to arrive at an appropriate value. This can raise issues of multiple hypothesis testing, although some methods, including the spatial scan statistic and Tango's maximised excess events test [64], are able to adjust for this while evaluating clustering at multiple scales. Results of spatial analyses can also suffer from lack of specificity. For example, in several studies of Legionnaires' disease, spatial methods identified areas most likely to be the source of the infection, but could not discriminate between potential sources that were close together $[40,57,58]$.

Another barrier to the effective use of spatial methods that is often cited is the expense of specialised GIS software and the need for trained personnel to operate it. Although some GIS programmes are available free of charge, the most commonly used was a commercial package, ArcGIS. However, it is also noteworthy that spatial scan statistics were the most frequently adopted analytic methods. Scan statistics can be implemented with relatively little training through SaTScan, a programme free to download from the Internet. This suggests a possible model for wider adoption of other more advanced techniques.

The results of our study point to a number of recommendations for improved practice and opportunities for further development of spatial methods. Given the potential utility of existing tools demonstrated here, under-use of these methods has doubtless resulted in missed opportunities for more effective real-time outbreak investigations. Public health officials must be supported to address this issue, and a useful first step would be development of protocols describing the application of appropriate analyses. Table 5 , which relates spatial methods to specific stages in outbreak investigations, provides a framework for this. Provision of training, for example through short courses, and interdisciplinary working with specialists in geographic analysis would also be beneficial to improve the skills base of the workforce.

The majority of studies identified in this review that used analytic methods described retrospective analysis of data collected during outbreaks. These reports demonstrated the potential utility of analytic methods, but will be of greater public health benefit when used in real time. Assembly of GIS databases in advance is essential to allow spatial analyses during prospective outbreak investigations. Improving data accessibility will save time during investigations, improve accuracy of analyses and prevent duplication of effort. Reports of analyses using spatial methods would also benefit from some degree of standardisation. For example, reporting of the sources and level of precision of spatial data would enable more accurate interpretation of the results by researchers not familiar with the study site. This could be achieved, for example, through extension of the Strengthening the Reporting of Observational Studies in Epidemiology (STROBE) statement with items specific to spatial data [65].

Finally, there is scope for development of new tools for analysis and visualisation of spatial data. A move towards web-based applications with user-friendly interfaces would be a natural progression, provided that these platforms included adequate training materials and data governance infrastructure. This would make spatial analyses more accessible to non-experts and could facilitate wider use of interactive displays of data and animations. The quantity and detail of geolocated data available to researchers is also increasing. GPS-enabled mobile devices and applications for self-reported or crowd-sourced information (for example sickweather [66], based on reports on social networks) have the potential to provide near real-time data including information on individuals' movements. Development of new analytic techniques will be needed to ensure that these data are effectively exploited and 
potential benefits are met. In the context of outbreak investigations, possible applications include contact tracing and improved estimation of exposure to environmental risk factors.

The primary limitation of this study was the challenge of designing the database search strategy. Although we employed a broad search which identified a large number of abstracts for screening, the number of studies identified here will inevitably be an underestimate of the outbreak investigations that used spatial methods. Our search will not have captured studies that used spatial methods but did not refer to them explicitly in the title, abstract, subject headings or MeSH terms. Restricting the search to articles published in academic journals also excluded reports in the grey literature. Inclusion of such reports would increase the number of investigations using spatial methods, but would be unlikely to reveal novel approaches or tools not identified here. Articles published since the database search was run at the end of 2013 are also not included in this study. Recent years have seen an increase in reports using spatial methods, probably due to increased availability of GIS software. This trend is likely to have continued, and recent publications will focus on current public health issues, for example the recent Ebola outbreak in West Africa.

There was also a possible publication bias in this study: spatial analyses may have been more likely to be presented in published reports if they were found to be useful. Concerns of breaching patient confidentiality could have further limited the number of studies that published maps. Nevertheless, the proportion of studies using spatial methods was very small, and even if our estimate is an order of magnitude too low, it would still represent less than $5 \%$ of the estimated total number of investigations published.

\section{Conclusion}

Investigations of outbreaks of infectious diseases require synthesis of information and expertise from a range of fields. Spatial analyses can make many valuable contributions, with simple maps alone providing fundamental insights about the distribution of cases. However, advancements in GIS technology and increasing availability of good quality spatial data provide an opportunity for development and implementation of more sophisticated tools. Adoption of these new techniques, and wider use of existing methods, has the potential to support more effective investigations and therefore limit the public health impacts of infectious disease outbreaks.

\section{Conflict of interest}

None declared.
Authors' contributions

$\mathrm{CS}$ and $\mathrm{ACH}$ designed the study. CS did the literature search, analyses and wrote the first draft. ACH, SLC, HF, MB and SL revised and edited the final report

\section{References}

1. Centers for Disease Control and Prevention (CDC). Mission, role and pledge. Atlanta; CDC. [Accessed: 25 Sep 2014]. Available from: http://www.cdc.gov/about/organization/mission.htm

2. GoodmanRA, BuehlerJW, KoplanJP. The epidemiologic field investigation: science and judgment in public health practice. Am J Epidemiol. 1990;132(1):9-16.PMID: 2356818

3. Snow J. On the Mode of Communication of Cholera. London: John Churchill; 1855.

4. World Health Organization (WHO). Foodborne disease outbreaks: guidelines for investigation and control. Geneva: WHO; 2008. Available from: http://www.who.int/foodsafety/ publications/foodborne_disease/outbreak_guidelines.pdf

5. Public Health England (PHE). Communicable disease outbreak management: operational guidance. London: PHE; 2014. Available from: https://www.gov.uk/government/uploads/ system/uploads/attachment_data/file/343723/12_8_2014_ CD Outbreak Guidance REandCT 2 2 .pdf

6. European Centre for Disease Prevention and Control (ECDC). Toolkit for investigation and response to food and waterborne disease outbreaks with an EU dimension. Stockholm: ECDC. [Accessed: 30 Sep 2014]. Available from: http://www.ecdc. europa.eu/en/healthtopics/food_and_waterborne_disease/ toolkit/Pages/index.aspx

7. Centers for Disease Control and Prevention (CDC). Multistate and nationwide foodborne outbreak investigations: a step-by step guide: Atlanta: CDC. [Accessed: 30 Sep 2014]. Available from: http://www.cdc.gov/foodsafety/outbreaks/investigatingoutbreaks/investigations/index.html

8. MooreDA, CarpenterTE. Spatial analytical methods and geographic information systems: use in health research and epidemiology.Epidemiol Rev. 1999;21(2):143-61. DOI: 10.1093/ oxfordjournals.epirev.a017993 PMID: 10682254

9. Pfeiffer DU, Robinson T, Stevenson M, Stevens KB, Rogers D, Clements AC. Spatial analysis in epidemiology. Oxford: Oxford University Press; 2008.

10. Bull M, Hall IM, Leach S, Robesyn E. The application of geographic information systems and spatial data during Legionnaires disease outbreak responses. Euro Surveill. 2012;17(49): $\mathrm{pii}=20331$.

11. HackertVH, van der HoekW, Dukers-MuijrersN, de BruinA, Al DahoukS, NeubauerH, et al. Q fever: single-point source outbreak with high attack rates and massive numbers of undetected infections across an entire region. Clin Infect Dis. 2012;55(12):1591-9. DOI: 10.1093/cid/cis734 PMID: 22918992

12. Guidelines for Investigating Clusters of Health Events. MMWR Recomm Rep. 1990;39(RR-11):1-23. PMID:2117247

13. CuzickJ, EdwardsR. Spatial clustering for inhomogeneous populations.J R Stat Soc.1990;52:73-104.

14. KulldorffM. A spatial scan statistic.Commun Stat Theory Methods. 1997;26(6):1481-96. DOI: 10.1080/03610929708831995

15. KulldorffM, HeffernanR, Hartman], AssunçãoRM, MostashariF. A space-time permutation scan statistic for the early detection of disease outbreaks.PLoS Med. 2005;2(3):e59. DOI: 10.1371/ journal.pmed.0020059

16. JacquezGM. Disease cluster statistics for space-time interaction. Stat Med. 1996;15(7-9):873-85. DOI: 10.1002/ (SICI)1097-0258(19960415)15:7/9<873::AID-SIM256>3.0.CO;2-U PMID: 8861156

17. European Centre for Disease Prevention and Control (ECDC). Outbreak investigations. Stockholm: ECDC. [Accessed: 15 Oct 2014]. Available from: https://wiki.ecdc.europa.eu/fem/w/ wiki/outbreak-investigations.aspx

18. UlugtekinN, AlkoyS, SekerDZ. Use of a geographic information system in an epidemiological study of measles in Istanbul.J Int Med Res. 2007;35(1):150-4. DOI: 10.1177/147323000703500117 PMID: 17408067

19. BarretoML. The dot map as an epidemiological tool: a case study of Schistosoma mansoni infection in an urban setting. Int J Epidemiol. 1993;22(4):731-41. DOI: 10.1093/ije/22.4.731 PMID: 8225750

20. RoquetD, DialloA, KodioB, DaffBM, FenechC, EtardJF. [Cholera epidemic in Senegal in 1995-1996: an example of geographic approach to health]. Sante. 1998;8(6):421-8. French. 
21. BarcellosC, SabrozaPC. Socio-environmental determinants of the leptospirosis outbreak of 1996 in western Rio de Janeiro: a geographical approach.Int J Environ Health Res. 2000;10(4):301-13. DOI: 10.1080/0960312002001500 PMID: 11260779

22. ParkinsonR, RajicA, JensonC. Investigation of an anthrax outbreak in Alberta in 1999 using a geographic information system.Can Vet J. 2003;44(4):315-8.PMID: 12715984

23. RivasAL, ChowellG, SchwagerSJ, FasinaFO, HoogesteijnAL, SmithSD, et al. Lessons from Nigeria: the role of roads in the geo-temporal progression of avian influenza $\left(\mathrm{H}_{5} \mathrm{~N}_{1}\right)$ virus. Epidemiol Infect. 2010;138(2):192-8. DOI: 10.1017/ So950268809990495 PMID: 19653927

24. AffolabiD, FaïhunF, SanoussiN, AnyoG, ShamputalC, RigoutsL, et al. Possible outbreak of streptomycin-resistant Mycobacterium tuberculosis Beijing in Benin. Emerg Infect Dis. 2009;15(7):1123-5. DOI: 10.3201/eid1507.080697 PMID: 19624936

25. RoyM, BenedictK, DeakE, KirbyMA, McNielJT, SicklerCJ, et al. A large community outbreak of blastomycosis in Wisconsin with geographic and ethnic clustering. Clin Infect Dis. 2013;57(5):655-62. DOI: 10.1093/cid/cit366 PMID: 23735332

26. South Wales Legionnaires Disease Outbreak Control Team,KeramarouM, EvansMR,. A community outbreak of Legionnaires' disease in South Wales, August-September 2010. Euro Surveill. 2010;15(42):pii=19691.PMID: 21034723

27. Bali S, Kar SS, Kumar S, Ratho RK, Dhiman RK, Kumar R. Hepatitis E epidemic with bimodal peak in a town of north India. Indian J Public Health. 2008;52(4):189-93, 99.

28. FitzpatrickG, WardM, EnnisO, JohnsonH, CotterS, CarrMJ, et al. Use of a geographic information system to map cases of measles in real-time during an outbreak in Dublin, Ireland, 2011. Euro Surveill. 2012;17(49):19-29.PMID: 23231894

29. KistemannT, DangendorfF, KrizekL, SahlHG, EngelhartS, ExnerM. GIS-supported investigation of a nosocomial Salmonella outbreak.Int J Hyg Environ Health. 2000;203(2):11726. DOI: 10.1078/S1438-4639(04)70016-4 PMID: 11109563

30. SasakiS, SuzukiH, IgarashiK, TambatambaB, MulengaP. Spatial analysis of risk factor of cholera outbreak for 2003-2004 in a peri-urban area of Lusaka, Zambia.Am J Trop Med Hyg. 2008;79(3):414-21.PMID: 18784235

31. NorströmM, PfeifferDU, JarpJ. A space-time cluster investigation of an outbreak of acute respiratory disease in Norwegian cattle herds.Prev Vet Med. 1999;47(1-2):107-19. DOI: 10.1016/S0167-5877(00)00159-8 PMID: 11018738

32. NygårdK, Werner-Johansen $\emptyset$, RønsenS, CaugantDA, Simonsen $\varnothing$, KanestrømA, et al. An outbreak of legionnaires disease caused by long-distance spread from an industrial air scrubber in Sarpsborg, Norway. Clin Infect Dis. 2008;46(1):619. DOI: $10.1086 / 524016$ PMID: 18171215

33. WallenstenA, MooreP, WebsterH, JohnsonC, van der BurgtG, PritchardG, et al. Q fever outbreak in Cheltenham, United Kingdom, in 2007 and the use of dispersion modelling to investigate the possibility of airborne spread. Euro Surveill. 2010;15(12):pii=19521.PMID: 20350497

34. Le ComberSC, RossmoDK, HassanAN, FullerDO, BeierJC. Geographic profiling as a novel spatial tool for targeting infectious disease control.Int J Health Geogr. 2011;10(1):35. DOI: 10.1186/1476-072X-10-35 PMID: 21592339

35. FèvreEM, ColemanPG, OdiitM, MagonaJW, WelburnSC, WoolhouseMEJ. The origins of a new Trypanosoma brucei rhodesiense sleeping sickness outbreak in eastern Uganda. Lancet. 2001;358(9282):625-8. DOI: 10.1016/S01406736(01)05778-6 PMID: 11530149

36. McKeeKT, ShieldsTM, JenkinsPR, ZenilmanJM, GlassGE. Application of a geographic information system to the tracking and control of an outbreak of shigellosis.Clin Infect Dis. 2000;31(3):728-33. DOI: 10.1086/314050 PMID: 11017823

37. KnoxEG. The detection of space-time interactions.J Appl Stat. 1964;13:24-30.

38. WhitePS, GrahamFF, HarteDJG, BakerMG, AmbroseCD, HumphreyARG. Epidemiological investigation of a Legionnaires' disease outbreak in Christchurch, New Zealand: the value of spatial methods for practical public health.Epidemiol Infect. 2013;141(4):789-99. DOI: 10.1017/ So950268812000994 PMID: 22697112

39. WongBCK, LeeN, LiY, ChanPKS, QiuH, LuoZ, et al. Possible role of aerosol transmission in a hospital outbreak of influenza. Clin Infect Dis. 2010;51(10):1176-83. DOI: $10.1086 / 656743$ PMID: 20942655

40. Hereford Legionnaires Outbreak Control Team,KirrageD, ReynoldsG, SmithGE, OlowokureB. Investigation of an outbreak of Legionnaires' disease: Hereford, UK 2003. Respir Med.
2007;101(8):1639-44. DOI: 10.1016/j.rmed.2006.11.026 PMID: 17513103

41. NygårdK, SchimmerB, Søbstad $\emptyset$, WaldeA, Tveitl, LangelandN, et al. A large community outbreak of waterborne giardiasisdelayed detection in a non-endemic urban area. BMC Public Health. 2006;6(1):141. DOI: 10.1186/1471-2458-6-141 PMID: 16725025

42. GubbelsS-M, KuhnKG, LarssonJT, AdelhardtM, EngbergJ, IngildsenP, et al. A waterborne outbreak with a single clone of Campylobacter jejuni in the Danish town of Køge in May 2010. Scand J Infect Dis. 2012;44(8):586-94. DOI: 10.3109/00365548.2012.655773 PMID: 22385125

43. NishaV, GadSS, SelvapandianD, SuganyaV, RajagopalV, SugantiP, et al. Geographical information system (GIS) in investigation of an outbreak. J Commun Dis. 2005;37(1):39-43 PMID: 16637399

44. AchesonP, McGivernM, FrankP, KunongaE, Simmsl, TayalS, et al. An ongoing outbreak of heterosexually-acquired syphilis across Teesside, UK. Int J STD AIDS. 2011;22(9):514-6. DOI: 10.1258/ijsa.2011.011008 PMID: 21890548

45. Luque FernándezMÁ, MasonPR, GrayH, BauernfeindA, FesseletJF, MaesP. Descriptive spatial analysis of the cholera epidemic 2008-2009 in Harare, Zimbabwe: a secondary data analysis.Trans R Soc Trop Med Hyg. 2011;105(1):38-45. DOI: 10.1016/j.trstmh.2010.10.001 PMID: 21075411

46. SarkarR, PrabhakarAT, ManickamS, SelvapandianD, RaghavaMV, KangG, et al. Epidemiological investigation of an outbreak of acute diarrhoeal disease using geographic information systems. Trans R Soc Trop Med Hyg. 2007;101(6):587-93. DOI: 10.1016/j.trstmh.2006.11.005 PMID: 17267000

47. HylandJM, HamletN, SaundersC, CoppolaJ, WattJ. Outbreak of Legionnaires' disease in West Fife: review of environmental guidelines needed.Public Health. 2008;122(1):79-83. DOI: 10.1016/j.puhe.2007.05.005 PMID: 17663011

48. RotelaC, FouqueF, LamfriM, SabatierP, IntroiniV, ZaidenbergM, et al. Space-time analysis of the dengue spreading dynamics in the 2004 Tartagal outbreak, Northern Argentina. Acta Trop. 2007;103(1):1-13. DOI: 10.1016/j.actatropica.2007.05.003 PMID 17603989

49. LuqueroFJ, BangaCN, RemartínezD, PalmaPP, BaronE, GraisRF. Cholera epidemic in Guinea-Bissau (2008): the importance of “place".PLoS ONE. 2011;6(5):e19005. DOI: 10.1371/journal. pone.0019005 PMID: 21572530

50. van der HoekW, van de KassteeleJ, BomB, de BruinA, DijkstraF, SchimmerB, et al. Smooth incidence maps give valuable insight into $Q$ fever outbreaks in The Netherlands. Geospat Health. 2012;7(1):127-34. DOI: 10.4081/gh.2012.111 PMID: 23242690

51. DavisGS, SevdalisN, DrumrightLN. Spatial and temporal analyses to investigate infectious disease transmission within healthcare settings.J Hosp Infect. 2014;86(4):227-43. DOI: 10.1016/j.jhin.2014.01.010 PMID: 24650720

52. European Food Safety Authority (EFSA), European Centre for Disease Prevention and Control (ECDC). The European Union summary report on trends and sources of zoonoses, zoonotic agents and food-borne outbreaks in 2013. EFSA Journal. 2015;13(1):3991. Available from: http://www.efsa.europa.eu/ en/efsajournal/pub/3991

53. Centers for Disease Control and Prevention (CDC). Surveillance for foodborne disease outbreaks, United States, 2012: Annual report. Atlanta: CDC; 2014 . Available from: http://www.cdc. gov/foodsafety/pdfs/foodborne-disease-outbreaks-annualreport-2012-508c.pdf

54. TanserFC, Le SueurD. The application of geographical information systems to important public health problems in Africa.Int J Health Geogr. 2002;1:4. DOI: 10.1186/1476-072X-1-4 PMID: 12537589

55. ChadeeDD, WilliamsFLR, KitronUD. Impact of vector control on a dengue fever outbreak in Trinidad, West Indies, in 1998. Trop Med Int Health. 2005;10(8):748-54. DOI: 10.1111/j.13653156.2005.01449.x PMID: 16045461

56. SowmyanarayananTV, MukhopadhyaA, GladstoneBP, SarkarR, KangG. Investigation of a hepatitis A outbreak in children in an urban slum in Vellore, Tamil Nadu, using geographic information systems.Indian J Med Res. 2008;128(1):32-7.PMID: 18820356

57. García-FulgueirasA, NavarroC, FenollD, GarcíaJ, GonzálezDiegoP, Jiménez-BuñualesT, et al. Legionnaires' disease outbreak in Murcia, Spain. Emerg Infect Dis. 2003;9(8):915-21. DOI: 10.3201/eido908.030337 PMID: 12967487

58. JansàJM, CaylàjA, FerrerD, GraciaJ, PelazC, SalvadorM, et al. An outbreak of Legionnaires' disease in an inner city district: importance of the first 24 hours in the investigation. Int J Tuberc Lung Dis. 2002;6(9):831-8.PMID: 12234140 
59. CarrR, WarrenR, TowersL, BartholomewA, DuggalHV, RehmanY, et al. Investigating a cluster of Legionnaires' cases: public health implications. Public Health. 2010;124(6):326-31. DOI: 10.1016/j.puhe.2010.03.001 PMID: 20483439

6o. BrownCM, NuortiPJ, BreimanRF, HathcockAL, FieldsBS, LipmanHB, et al. A community outbreak of Legionnaires' disease linked to hospital cooling towers: an epidemiological method to calculate dose of exposure. Int J Epidemiol. 1999;28(2):353-9. DOI: 10.1093/ije/28.2.353 PMID: 10342703

61. Bhaduri B. Encyclopedia of GIS. New York: Springer; 2008.

62. MartinD. Directions in Population GIS.Geogr Compass. 2011;5(9):655-65. DOI: 10.1111/j.1749-8198.2011.00440.x

63. NguyenTM, IlefD, JarraudS, RouilL, CampeseC, CheD, et al. A community-wide outbreak of legionnaires disease linked to industrial cooling towers--how far can contaminated aerosols spread? J Infect Dis. 2006;193(1):102-11. DOI: 10.1086/498575 PMID: 16323138

64. TangoT. A test for spatial disease clustering adjusted for multiple testing. Stat Med. 2000;19(2):191-204. DOI: 10.1002 (SICI)1097-0258(20000130)19:2〈191::AID-SIM281〉3.0.CO;2-Q PMID: 10641024

65. von ElmE, AltmanDG, EggerM, PocockSJ, GøtzschePC, VandenbrouckeJP, et al. The Strengthening the Reporting of Observational Studies in Epidemiology (STROBE) statement: guidelines for reporting observational studies. Prev Med. 2007;45(4):247-51. DOI: 10.1016/j.ypmed.2007.08.012 PMID: 17950122

66. Sickweather. Baltimore: Sickweather Inc. [Accessed: 15 Oct 2014]. Available from: http://www.sickweather.com/

67. BocciaD, OliverCI, CharlettA, BennettS, OrrH, SarangiJ, et al. Outbreak of a new Salmonella phage type in South West England: alternative epidemiological investigations are needed. Commun Dis Public Health. 2004;7(4):339-43.PMID: 15779803

68. Neira-MunozE, OkoroC, McCarthyND. Outbreak of waterborne cryptosporidiosis associated with low oocyst concentrations. Epidemiol Infect. 2007;135(7):1159-64. DOI: 10.1017/ S0950268807008503 PMID: 17445321

69. SansonRL, GlosterJ, BurginL. Reanalysis of the start of the UK 1967 to 1968 foot-and-mouth disease epidemic to calculate airborne transmission probabilities.Vet Rec. 2011;169(13):336 DOI: 10.1136/vr.d4401 PMID: 21846685

70. Manfredi SelvaggiT, RezzaG, ScagnelliM, RigoliR, RassuM, De LallaF, et al. Investigation of a Q-fever outbreak in northern Italy. Eur J Epidemiol. 1996;12(4):403-8. DOI: 10.1007/ BFo0145305 PMID: 8891546

71. OrsiA, AlicinoC, PatriaAG, ParodiV, CarloniR, TurelloV, et al. Epidemiological and molecular approaches for management of a measles outbreak in Liguria, Italy. J Prev Med Hyg. 2010;51(2):67-72.PMID: 21155408

72. VaraniS, CagarelliR, MelchiondaF, AttardL, SalvadoriC, FinarelliAC, et al. Ongoing outbreak of visceral leishmaniasis in Bologna Province, Italy, November 2012 to May 2013. Euro Surveill. 2013;18(29):20530. DOI: 10.2807/1560-7917. ES2013.18.29.20530 PMID: 23929116

73. AbellánJJ, Martínez-BeneitoMA, ZurriagaO, JorquesG, Ferrándiz], López-QuílezA. [Point processes as a tool for analyzing possible sources of contamination]. Gac Sanit. 2002;16(5):445-9. Spanish.PMID: 12372192

74. SchimmerB, Ter ScheggetR, WegdamM, ZüchnerL, de BruinA, SchneebergerPM, et al. The use of a geographic information system to identify a dairy goat farm as the most likely source of an urban Q-fever outbreak. BMC Infect Dis. 2010;10(1):69. DOI: 10.1186/1471-2334-10-69 PMID: 20230650

75. Lai P-c, Kwong K-h. Spatial Analysis of the 2008 Influenza Outbreak of Hong Kong. Computational Science and Its Applications - Iccsa 2010, Pt 1, Proceedings. Lecture Notes in Computer Science. 60162010. p. 374-88.

76. LaiPC, WongCM, HedleyAJ, LoSV, LeungPY, KongJ, et al. Understanding the spatial clustering of severe acute respiratory syndrome (SARS) in Hong Kong. Environ Health Perspect. 2004;112(15):1550-6. DOI: 10.1289/ehp.7117 PMID: 15531441

77. Sze-ToGN, ChaoCYH. Use of risk assessment and likelihood estimation to analyze spatial distribution pattern of respiratory infection cases. Risk Anal. 2011;31(3):351-69. DOI: 10.1111/j.1539-6924.2010.01525.x PMID: 21039710

78. YulTS, WongTW, ChiuYL, LeeN, LiY. Temporal-spatial analysis of severe acute respiratory syndrome among hospital inpatients. Clin Infect Dis. 2005;40(9):1237-43. DOI: 10.1086/428735 PMID: 15825024

79. SahaT, MurhekarM, HutinYJ, RamamurthyT. An urban, waterborne outbreak of diarrhoea and shigellosis in a district town in eastern India.Natl Med J India. 2009;22(5):237-9.PMID: 20334044
80. FangL-Q, LiX-L, LiuK, LiY-J, YaoH-W, LiangS, et al. Mapping spread and risk of avian influenza $A\left(\mathrm{H}_{7} \mathrm{~N} 9\right)$ in China. Sci Rep. 2013;3:2722. DOI: 10.1038/srepo2722 PMID: 24072008

81. LiangW, McLawsML, LiuM, MiJ, ChanDKY. Hindsight: a reanalysis of the severe acute respiratory syndrome outbreak in Beijing.Public Health. 2007;121(10):725-33. DOI: $10.1016 / j$. puhe.2007.02.023 PMID: 17555781

82. AliM, WagatsumaY, EmchM, BreimanRF. Use of a geographic information system for defining spatial risk for dengue transmission in Bangladesh: role for Aedes albopictus in an urban outbreak.Am J Trop Med Hyg. 2003;69(6):634-40.PMID: 14740881

83. TenzinSB, SharmaB, DhandNK, TimsinaN, WardMP. Reemergence of rabies in Chhukha district, Bhutan, 2008. Emerg Infect Dis. 2010;16(12):1925-30. DOI: 10.3201/ eid1612.100958 PMID: 21122223

84. NishiguchiA, KobayashiS, OuchiY, YamamotoT, HayamaY, TsutsuiT. Spatial analysis of low pathogenic $\mathrm{H}_{5} \mathrm{~N}_{2}$ avian influenza outbreaks in Japan in 2005.J Vet Med Sci. 2009;71(7):979-82. DOI: 10.1292/jvms.71.979 PMID: 19652489

85. SiddiquiFJ, BhuttoNS, von SeidleinL, Khurraml, RasoolS, AliM, et al. Consecutive outbreaks of Vibrio cholerae 0139 and V. cholerae $\mathrm{O}_{1}$ cholera in a fishing village near Karachi, Pakistan. Trans R Soc Trop Med Hyg. 2006;100(5):476-82. DOI: 10.1016/j. trstmh.2005.07.019 PMID: 16443247

86. Miranda ME, Yoshikawa Y, Manalo DL, Calaor AB, Miranda NL, Cho F, et al. Chronological and spatial analysis of the 1996 Ebola Reston virus outbreak in a monkey breeding facility in the Philippines. Exp Anim. 2002;51(2):173-9.

87. LeH, PoljakZ, DeardonR, DeweyCE. Clustering of and risk factors for the porcine high fever disease in a region of Vietnam.Transbound Emerg Dis. 2012;59(1):49-61. DOI: 10.1111/j.1865-1682.2011.01239.x PMID: 21722329

88. AddissDG, DavisJP, LaVentureM, WandPJ, HutchinsonMA, McKinneyRM. Community-acquired Legionnaires' disease associated with a cooling tower: evidence for longer-distance transport of Legionella pneumophila.Am J Epidemiol. 1989;130(3):557-68.PMID: 2764000

89. BlondinN, BaumgardnerDJ, MooreGE, GlickmanLT. Blastomycosis in indoor cats: suburban Chicago, Illinois, USA. Mycopathologia. 2007;163(2):59-66. DOI: 10.1007/s11046-0060090-1 PMID: 17262169

90. ChungWM, BusemanCM, JoynerSN, HughesSM, FombyTB, LubyJP, et al. The 2012 West Nile encephalitis epidemic in Dallas, Texas. JAMA. 2013;310(3):297-307. DOI: 10.1001/ jama.2013.8267 PMID: 23860988

91. MongohMN, DyerNW, StoltenowCL, KhaitsaML. Risk factors associated with anthrax outbreak in animals in North Dakota, 2005: a retrospective case-control study.Public Health Rep. 2008;123(3):352-9.PMID: 19006977

92. PfisterJR, ArcherJR, HersilS, BoersT, ReedKD, MeeceJK, et al. Non-rural point source blastomycosis outbreak near a yard waste collection site. Clin Med Res. 2011;9(2):57-65. DOI: 10.3121/cmr.2010.958 PMID: 20974888

93. BowieWR, KingAS, WerkerDH, Isaac-RentonJL, BellA, EngSB et al. Outbreak of toxoplasmosis associated with municipal drinking water. Lancet. 1997;350(9072):173-7. DOI: 10.1016/ S0140-6736(96)11105-3 PMID: 9250185

94. EppT, ArgueC, WaldnerC, Berke0. Spatial analysis of an anthrax outbreak in Saskatchewan, 2006.Can Vet J. 2010;51(7):743-8.PMID: 20885827

95. PasmaT. Spatial epidemiology of an $\mathrm{H}_{3} \mathrm{~N}_{2}$ swine influenza outbreak.Can Vet J. 2008;49(2):167-76.PMID: 18309747

96. MorrisonAC, GetisA, SantiagoM, Rigau-PerezJG, ReiterP. Exploratory space-time analysis of reported dengue cases during an outbreak in Florida, Puerto Rico, 1991-1992.Am J Trop Med Hyg. 1998;58(3):287-98.PMID: 9546405

97. ChadeeDD, LeeR, FerdinandA, PrabhakarP, ClarkeD, JacobB. Meningococcal meningitis outbreak in Trinidad, 1998.European Journal of General Medicine.2006;3(2):49-53.

98. Bartels SA, Greenough PG, Tamar M, VanRooyen MJ. Investigation of a cholera outbreak in Ethiopia's Oromiya Region. Disaster med. 2010;4(4):312-7.

99. BessongPO, OdiyoJO, MusekeneJN, TessemaA. Spatial distribution of diarrhoea and microbial quality of domestic water during an outbreak of diarrhoea in the Tshikuwi community in Venda, South Africa.J Health Popul Nutr. 2009;27(5):652-9. DOI: 10.3329/jhpn.v27i5.3642 PMID: 19902801

100. AnguloJJ, PederneirasCA, SakumaME, TakigutiCK, MegaleP. Contour mapping of the temporal-spatial progression of a contagious disease.Bull Soc Pathol Exot Filiales. 1979;72(4):374-85.PMID: 535118

101. de MouraL, Bahia-OliveiraLMG, WadaMY, JonesJL, TuboiSH, CarmoEH, et al. Waterborne toxoplasmosis, Brazil, from 
field to gene. Emerg Infect Dis. 2006;12(2):326-9. DOI: 10.3201/eid1202.041115 PMID: 16494765

102. PassosAD, Castro e SilvaAA, FerreiraAH, Maria e SilvaJ, MonteiroME, SantiagoRC. [Rabies epizootic in the urban area of Ribeirão Preto, São Paulo, Brazil]. Cad Saude Publica. 1998;14(4):735-40. Portuguese. DOI: 10.1590/ S0102-311X1998000400015 PMID: 9878906

103. RivasAL, SmithSD, SullivanPJ, GardnerB, AparicioJP, HoogesteijnAL, et al. Identification of geographic factors associated with early spread of foot-and-mouth disease. Am J Vet Res. 2003;64(12):1519-27. DOI: 10.2460/ ajvr.2003.64.1519 PMID: 14672431

104. FirestoneSM, WardMP, ChristleyRM, DhandNK. The importance of location in contact networks: Describing early epidemic spread using spatial social network analysis.Prev Vet Med. 2011;102(3):185-95. DOI: 10.1016/j. prevetmed.2011.07.006 PMID: 21852007

105. WaldronLS, FerrariBC, Cheung-Kwok-SangC, BeggsPJ,

StephensN, PowerML. Molecular epidemiology and spatial distribution of a waterborne cryptosporidiosis outbreak in Australia.Appl Environ Microbiol. 2011;77(21):7766-71. DOI: 10.1128/AEM.00616-11 PMID: 21908623

106. Turcios-RuizRM, AxelrodP, St JohnK, BullittE, DonahueJ, RobinsonN, et al. Outbreak of necrotizing enterocolitis caused by norovirus in a neonatal intensive care unit. J Pediatr. 2008;153(3):339-44. DOI: 10.1016/j. jpeds.2008.04.015 PMID: 18534621 